\title{
Effect of delamination on the stochastic natural frequencies of composite laminates
}

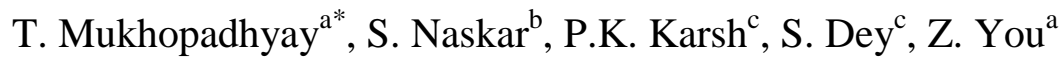 \\ ${ }^{a}$ Department of Engineering Science, University of Oxford, Oxford, UK \\ ${ }^{\mathrm{b}}$ School of Engineering, University of Aberdeen, Aberdeen, UK \\ ${ }^{\mathrm{c}}$ Mechanical Engineering Department, National Institute of Technology Silchar, India \\ "Corresponding author's e-mail: tanmoy.mukhopadhyay@eng.ox.ac.uk
}

\begin{abstract}
The coupled effect of manufacturing uncertainty and a critical service-life damage condition (delamination) is investigated on the natural frequencies of laminated composite plates. In general, delamination is an unavoidable phenomenon in composite materials encountered often in real-life operating conditions. We have focused on the characterization of dynamic responses of composite plates considering source-uncertainty in the material and geometric properties along with various single and multiple delamination scenarios. A hybrid high dimensional model representation based uncertainty propagation algorithm coupled with layer-wise stochastic finite element model of composites is developed to achieve computational efficiency. The finite element formulation is based on Mindlin's theory considering transverse shear deformation. Numerical results are presented for the stochastic natural frequencies of delaminated composites along with a comprehensive deterministic analysis. Further, an inevitable effect of noise is induced in the surrogate based analysis to explore the effect of various errors and epistemic uncertainties involved with the system.
\end{abstract}

Keywords: delamination; manufacturing uncertainty; laminated composite plate; surrogate based finite element method

\section{Introduction}

Laminated composites have gained preference in various engineering applications such as aerospace, naval, automobile, micro-electro-mechanical-systems (MEMS) and civil structures due to high strength and stiffness with weight-sensitivity, increased toughness, mechanical damping, as well as tailoring of structural properties. In the recent year, wide application of composite materials has drawn an increased attention to its operational reliability and safety. The exhaustive usage of such structures has warranted the detail understanding of damage modes and their consequences in global structural responses. One of the most 
significant setbacks of fibre-reinforced polymer composites is the propensity to onset, growth and propagation of delamination. In other words, one of the principle modes of failure in laminated composites is the delamination or separation of layers along the interfaces. In general, delamination occurs due unaccounted tension and shear developed at inter-laminar zones due to various factors such as free edge effect, discontinuities in structural elements, localized disturbances during manufacturing and operating conditions. The presence of inter-laminar debonding or delamination is often laid buried between the layers as it is hidden from superficial visual inspection. Subsequently the growth and propagation of delamination in conjunction with other modes of damage also remain unaccounted whereas it may reduce stiffness of the structure drastically leading to failure and instability of the structure.

Besides delamination in composites, these advanced materials are susceptible to various forms of source-uncertainties in material and structural attributes due to complex manufacturing process (such as intralaminate voids and excess matrix voids, excess resin between plies, incomplete curing of resin, porosity, variations in lamina thickness, fibre orientation and fibre properties) and complicated design requirements. Such uncertainties affect the global structural responses significantly. The coupled effect of delamination and inevitable source-uncertainties can drastically influence the structural responses computed based on deterministic assumptions.

Fundamental principles of stochastic mechanics are required to understand the probabilistic dynamic behavior of delaminated composites. It involves significant challenges to apply the original concepts developed for isotropic materials to laminated composites where material and geometric anisotropy prevails. Hence it is essential to investigate the complexities arising from unknown sources initiating the debonding of constitutive layers and its uncertain means of propagation. The free vibration characteristics of delaminated composites can show drastic fluctuations from the computed deterministic values due to randomness in material and geometric properties and damages incurred during the service-life depending on number, size or shape and location of delamination. Moreover, due to the involvement of random system properties, the vibration characteristics of such structures with delamination can behave differently in different modes.

A plenty of research work is reported on deterministic free vibration analysis of laminated composite plates and shells [1-10]. The aspect of delamination in composites has also received adequate attention in the deterministic domain [11-39]. Stochastic analysis of composite and sandwich structures considering sourceuncertainty is found to be studied by many researchers including the aspects of multi-scale analysis, 
optimization and reliability assessment [40-59]. However, the compound effect of delamination and sourceuncertainty has not been investigated yet for the dynamic responses of composite structures. In this paper, we aim to investigate this coupled effect on the natural frequencies of composite plates.

A careful review of literature concerning uncertainty quantification in composites shows two prominent approaches: perturbation based approach and Monte Carlo simulation based approach. The major drawback in a perturbation based approach can be identified as the requirement of intensive analytical derivation and lack of the ability to obtain complete probabilistic description of the response quantities. Moreover, this approach is valid only for a low degree of stochasticity in the input parameters. A Monte Carlo simulation (MCS) approach for uncertainty quantification does not have these critical lacunas. But the MCS approach is computationally very demanding because of the requirement of carrying out large number $\left(\sim 10^{4}\right)$ of repetitive simulations corresponding to a random set of input parameters. For the analyses of composite structures including the effect of delamination, even one such simulation is normally very computationally intensive and time consuming. In such situation the panacea is a surrogate based Monte Carlo simulation [6064], which is adopted in this study.

In the uncertainty quantification of responses by employing surrogate based approach, the original finite element model is replaced by an efficient pseudo simulation model, which is effective but economical. The surrogate models get necessary information about the nature of the response outputs by algorithmically selected design points drawn from the entire domain. An efficient hybrid high dimensional model representation (HDMR) [65-66] based uncertainty propagation algorithm coupled with layer-wise stochastic finite element (FE) model of the delaminated composites is developed in this paper for the stochastic free vibration analysis. The optimal design points are drawn from a pseudo random Sobol sequence [67]. In this context, another source of uncertainty needs be accounted in the analysis. The information acquired from the selected design points (input-output dataset) for forming surrogate models is a second source of uncertainty besides the conventional source-uncertainties in material and geometric parameters (refer to Figure 1). In the present study, simulated noise is introduced to account for such second source of uncertainty that can be tantamount to incorporating measurement error of responses, modelling and simulation error and other epistemic uncertainties involved in the system. To the best of authors' knowledge, the present paper is the first attempt for a surrogate based dynamic analysis of delaminated composite plates (refer to Figure 2) coupled with random material and geometric properties including the effect of inevitable noise. After the 


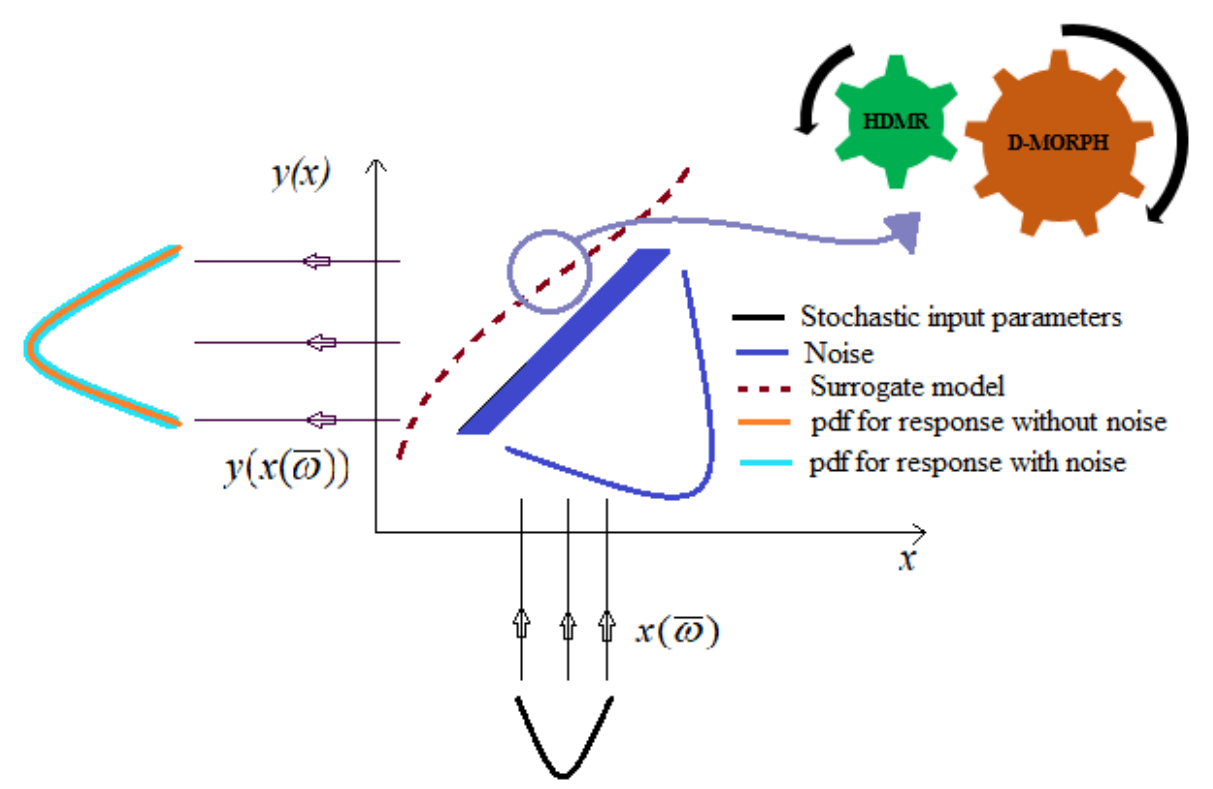

Fig. 1 Surrogate based stochastic analysis under the influence of noise

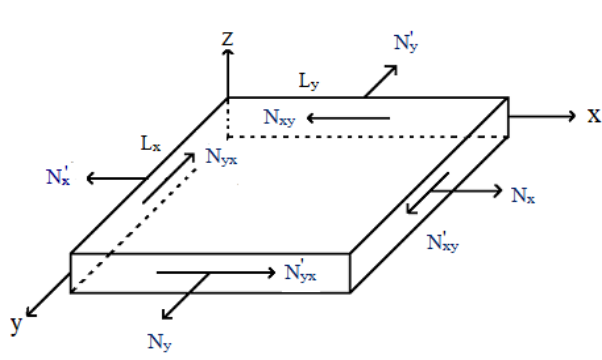

(a)

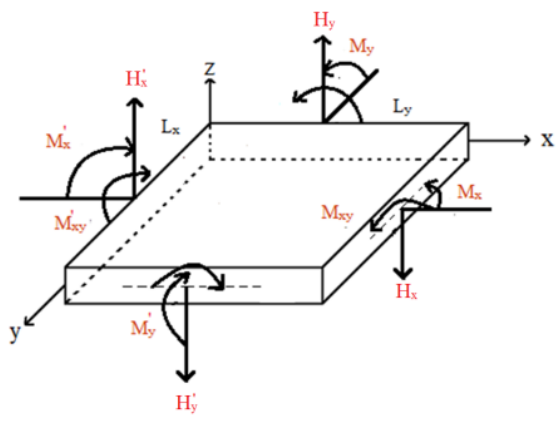

(b)

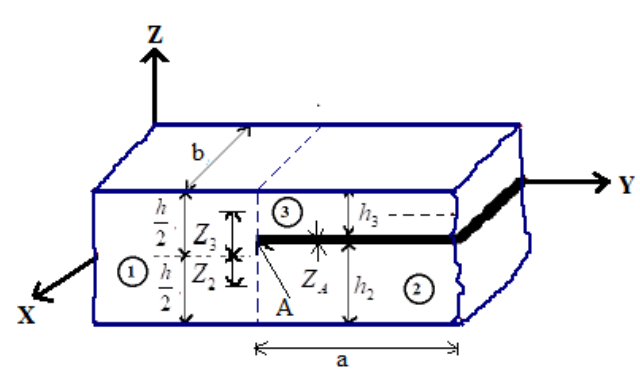

(c)

Fig. 2 (a, b) Composite plate showing forces and moments (c) Delamination in composite plates at crack tip

introduction section, this paper is organized as, section 2: brief description of the mathematical model for stochastic dynamic analysis of delaminated composite plates; section 3: Hybrid HDMR based FE algorithm for layer-wise stochastic modelling of delaminated composites including the effect of noise (detail mathematical formulation of HDMR based surrogate modelling is provided as APPENDIX); section 4: results and discussion; section 5: summary and perspective; section 6: conclusion.

\section{Stochastic dynamics of delaminated composite plates}

In the present article, a composite cantilever plates with thickness ' $h$ ' with length ' $L$ ' and width ' $b$ ' is considered as shown in Figure 2. According to the first-order shear deformation theory, the displacement field of the plates is described by 


$$
\begin{aligned}
& u(x, y, z)=u^{\prime}(x, y)-z \theta_{x}(x, y) \\
& v(x, y, z)=v^{\prime}(x, y)-z \theta_{y}(x, y) \\
& w(x, y, z)=w^{\prime}(x, y)=w(x, y)
\end{aligned}
$$

where, $u^{\prime}, v^{\prime}$ and $w^{\prime}$ denotes displacements corresponding to the reference planes, while $\theta_{x}$ and $\theta_{y}$ represents $\mathrm{X}$ and $\mathrm{y}$ axes rotations respectively. Each fibre of laminae is oriented at an arbitrary angle ' $\theta$ ' about the $\mathrm{x}$-axis. For the shell, the constitutive equations is given by [68]

$$
\{F\}=[D(\bar{\omega})]\{\varepsilon\}
$$

where $(\bar{\omega})$ is a typical representation of stochasticity. Here the force resultant is given by

$$
\begin{aligned}
& \{F\}=\left\{N(\bar{\omega})_{x}, N(\bar{\omega})_{y}, N(\bar{\omega})_{x y}, M(\bar{\omega})_{x}, M(\bar{\omega})_{y}, M(\bar{\omega})_{x y}, H(\bar{\omega})_{x}, H(\bar{\omega})_{y}\right\}^{T} \\
& \{F\}=\left[\int_{-h / 2}^{h / 2}\left\{\sigma_{x}, \quad \sigma_{y}, \quad \tau_{x y}, \quad \sigma_{x z}, \quad \sigma_{y z}, \quad \tau_{x y}, \quad \tau_{x z}, \tau_{y z}\right\} d z\right]^{T}
\end{aligned}
$$

The strain vector can be expressed as

$$
\{\varepsilon\}=\left\{\varepsilon_{x}, \varepsilon_{y}, \varepsilon_{x y}, k_{x}, k_{y}, k_{x y}, \gamma_{x z}, \gamma_{y z}\right\}^{T}
$$

The elements of elastic stiffness matrix $[D(\bar{\omega})]$ is given by

$$
\begin{gathered}
{\left[A_{i j}(\bar{\omega}), \quad B_{i j}(\bar{\omega}), \quad D_{i j}(\bar{\omega})\right]=\sum_{k=1}^{n} \int_{z_{k-1}}^{z_{k}}\left[\left\{\bar{H}_{i j}(\bar{\omega})\right\}_{o n}\right]_{k} \quad\left[\begin{array}{lll}
1, & z, & z^{2}
\end{array}\right] d z \quad i, j=1,2,6} \\
{\left[S_{i j}(\bar{\omega})\right]=\sum_{k=1}^{n} \int_{z_{k-1}}^{z_{k}} \alpha_{s}\left[\bar{H}(\bar{\omega})_{i j}\right]_{k} d z \quad i, j=4,5}
\end{gathered}
$$

The parameter $\alpha_{s}$ represents the shear correction factor (in the present study $\alpha_{s}=5 / 6$ ) and $\left[\bar{H}(\bar{\omega})_{i j}\right]$ denotes off-axis elastic constant matrix elements and expressed by

$$
\begin{aligned}
& {\left[\bar{H}(\bar{\omega})_{i j}\right]_{o f f}=\left[T_{1}(\bar{\omega})\right]^{-1}\left[\bar{H}(\bar{\omega})_{i j}\right]_{o n}\left[T_{1}(\bar{\omega})\right]^{-T} \quad \text { for } i, j=1,2,6} \\
& {\left[\bar{H}(\bar{\omega})_{i j}\right]_{o f f}=\left[T_{2}(\bar{\omega})\right]^{-1}\left[\bar{H}(\bar{\omega})_{i j}\right]_{o n}\left[T_{2}(\bar{\omega})\right]^{-T} \quad \text { for } i, j=4,5} \\
& {\left[T_{1}(\bar{\omega})\right]=\left[\begin{array}{ccc}
c^{2} & d^{2} & 2 c d \\
d^{2} & c^{2} & -2 c d \\
-c d & c d & c^{2}-d^{2}
\end{array}\right] \text { and } \quad\left[T_{2}(\bar{\omega})\right]=\left[\begin{array}{cr}
c & -d \\
d & c
\end{array}\right]}
\end{aligned}
$$

in which $c=\operatorname{Sin} \theta(\bar{\omega})$ and $d=\operatorname{Cos} \theta(\bar{\omega})$, wherein $\theta(\bar{\omega})$ is random fibre orientation angle.

$\left[H_{i j}(\bar{\omega})\right]_{o n}=\left[\begin{array}{ccc}H_{11} & H_{12} & 0 \\ H_{12} & H_{22} & 0 \\ 0 & 0 & H_{66}\end{array}\right]$ for $i, j=1,2,6 \quad\left[\bar{H}_{i j}(\bar{\omega})\right]_{o n}=\left[\begin{array}{cc}H_{44} & H_{45} \\ H_{45} & H_{55}\end{array}\right]$ for $i, j=4,5$

Where 


$$
H_{11}=\frac{E_{1}}{1-v_{12} v_{21}}, H_{22}=\frac{E_{2}}{1-v_{12} v_{21}}, H_{12}=\frac{v_{12} E_{2}}{1-v_{12} v_{21}}, H_{66}=G_{12}, H_{44}=G_{23} \text { and } H_{55}=G_{13}
$$

In the FE formulation, an isoparametric quadratic element with 64 elements and 225 nodes is considered in this paper and each node has five degrees of freedom (DOF) (three translations and two rotations). For composite plate the mass per unit area is given by

$$
P(\bar{\omega})=\sum_{k=1}^{n} \int_{z_{k-1}}^{z_{k}} \rho(\bar{\omega}) d z
$$

The mass matrix is given by

$$
[M(\bar{\omega})]=\int_{V o l}[N(\bar{\omega})][P(\bar{\omega})][N(\bar{\omega})] d(v o l)
$$

The stiffness matrix can be expressed by

$$
[K(\bar{\omega})]=\int_{-1}^{1} \int_{-1}^{1}[B(\bar{\omega})]^{T}[D(\bar{\omega})][B(\bar{\omega})] d \varsigma d \chi
$$

Lagrange's equation and Hamilton's principle [69] are employed to define the equation of motion for the free vibration system having $n$ DOF and given by

$$
[M(\bar{\omega})][\ddot{\delta}]+[K(\bar{\omega})]\{\delta\}=0
$$

where $[M(\bar{\omega})]$ represents mass matrix and $[K(\bar{\omega})]$ represents elastic stiffness matrix, while $\{\delta\}$ represents the vector of generalized coordinates. Mindlin's theory is employed to derive the governing equations for transverse shear deformation. In the free vibration analysis QR iteration algorithm is applied to determine the stochastic natural frequencies $\left[\omega_{n}(\bar{\omega})\right]$ by solving standard eigenvalue problem [70].

The cross-sectional view of delaminated composite crack tip is illustrated in Figure 2 (multipoint constraint - delamination model), in which a common node is formed by the nodes of three plate elements. The plate element 1 with thickness $h$ illustrates the undelaminated portion, while plate elements 2 and 3 show the delaminated portion. Delamination occurs at the interface of the plate element 2 and 3, where $h_{2}$ and $h_{3}$ are the thicknesses of the elements 2 and 3, respectively. Before application of constraints condition, the elements 1,2 and 3 are free to deform. At the crack tip, the nodal displacements of elements 2 and 3 can be given by [71]

$$
\begin{aligned}
& u_{j}=u_{j}^{\prime}-\left(z-z_{j}^{\prime}\right) \theta_{x j} \\
& v_{j}=v_{j}^{\prime}-\left(z-z_{j}^{\prime}\right) \theta_{y j} \\
& w_{j}=w_{j}^{\prime} \quad(\text { where, } \mathrm{j}=2,3)
\end{aligned}
$$


where $u_{j}^{\prime}, v_{j}^{\prime}$ and $w_{j}^{\prime}$ represents the mid-plane displacements in the $\mathrm{x}, \mathrm{y}$ and $\mathrm{z}$ direction respectively. $z_{j}^{\prime}$ represents the z-coordinate of mid-plane of element $\mathrm{j}$ while $\theta_{x}$ and $\theta_{y}$ denotes rotations about $\mathrm{x}$ and $\mathrm{y}$ axes, respectively. For the element 1 also the given equation is valid. The common node have relationship for transverse displacements and rotations as

$$
\begin{gathered}
w_{1}=w_{2}=w_{3}=w \\
\theta_{x 1}=\theta_{x 2}=\theta_{x 3}=\theta_{x} \\
\theta_{y 1}=\theta_{y 2}=\theta_{y 3}=\theta_{y}
\end{gathered}
$$

At the crack tip, in-plane displacements have same magnitude for all the three elements and they have relationship as

$$
\begin{aligned}
& u_{2}^{\prime}=u_{1}^{\prime}-z_{2}^{\prime} \theta_{x} \\
& v_{2}^{\prime}=v_{1}^{\prime}-z_{2}^{\prime} \theta_{y} \\
& u_{3}^{\prime}=u_{1}^{\prime}-z_{2}^{\prime} \theta_{x} \\
& v_{3}^{\prime}=v_{1}^{\prime}-z_{3}^{\prime} \theta_{y}
\end{aligned}
$$

where $u_{1}^{\prime}$ denotes the displacement of the element 1 at the mid-plane. Equations (13) and (14) are the multipoint constraint equations, which have relationship for the nodal displacements and rotations of elements at the crack tip. These equations satisfy the compatibility equations of displacement and rotations and these equations are employed for the finite element formulation in the present study. The mid-plane strains between elements 2 and 3 have relationship as,

$$
\left\{\varepsilon^{\prime}\right\}_{j}=\left\{\varepsilon^{\prime}\right\}_{1}+z_{j}^{\prime}\{k\}(\text { where } \mathrm{j}=2,3)
$$

Where $\left\{\varepsilon^{\prime}\right\}$ denotes the normal strain vector at mid-plane and $\{k\}$ denotes the curvature vector. For elements 1, 2 and 3 curvature vector have same value at the crack tip. This equation is the special case for element 1 when $z_{1}^{\prime}$ is equal to zero. For the element 2 and 3, in-plane stress-resultants $\{N(\bar{\omega})\}$ and moment resultants $\{M(\bar{\omega})\}$ are expressed by

$$
\begin{gathered}
\{N(\bar{\omega})\}_{j}=[A(\bar{\omega})]_{j}\left\{\varepsilon^{\prime}\right\}_{1}+\left(z_{j}^{\prime}[A(\bar{\omega})]_{j}+[B(\bar{\omega})]_{j}\{k\} \quad(\text { where } \mathrm{j}=2,3)\right. \\
\{M(\bar{\omega})\}_{j}=[B(\bar{\omega})]_{j}\left\{\varepsilon^{\prime}\right\}_{1}+\left(z_{j}^{\prime}[B(\bar{\omega})]_{j}+[D(\bar{\omega})]_{j}\{k\} \quad(\text { where } \mathrm{j}=2,3)\right. \\
\{H(\bar{\omega})\}_{j}=\left[A(\bar{\omega})^{*}\right]_{j}\{y\} \quad(\text { where } \mathrm{j}=2,3)
\end{gathered}
$$

In other form, 


$$
\begin{aligned}
& \left\{\begin{array}{l}
N(\bar{\omega})_{x} \\
N(\bar{\omega})_{y} \\
N(\bar{\omega})_{x y}
\end{array}\right\}=\sum_{k=1}^{n=8}\left[H(\bar{\omega})_{i j}\left\{\int_{z_{k-1}}^{z_{k}}\left\{\begin{array}{l}
\varepsilon_{x}^{\prime} \\
\varepsilon_{y}^{\prime} \\
\gamma_{x y}^{\prime}
\end{array}\right\} d z+\int_{z_{k-1}}^{z_{k}}\left\{\begin{array}{l}
k_{x} \\
k_{y} \\
k_{x y}
\end{array}\right\} z d z+z_{j}^{\prime} \int_{z_{k-1}}^{z_{k}}\left\{\begin{array}{l}
k_{x} \\
k_{y} \\
k_{x y}
\end{array}\right\} d z\right\} \quad \ldots i, j=1,2,6\right. \\
& \left\{\begin{array}{l}
M(\bar{\omega})_{x} \\
M(\bar{\omega})_{y} \\
M(\bar{\omega})_{x y}
\end{array}\right\}=\sum_{k=1}^{n=8}\left[H(\bar{\omega})_{i j}\left\{\int_{z_{k-1}}^{z_{k}}\left\{\begin{array}{l}
\varepsilon_{x}^{\prime} \\
\varepsilon_{y}^{\prime} \\
\gamma_{x y}^{\prime}
\end{array}\right\} z d z+\int_{z_{k-1}}^{z_{k}}\left\{\begin{array}{l}
k_{x} \\
k_{y} \\
k_{x y}
\end{array}\right\} z^{2} d z+z_{j}^{\prime} \int_{z_{k-1}}^{z_{k}}\left\{\begin{array}{l}
k_{x} \\
k_{y} \\
k_{x y}
\end{array}\right\} z d z\right\} \quad \ldots i, j=1,2,6\right. \\
& \left\{\begin{array}{l}
H(\bar{\omega})_{x} \\
H(\bar{\omega})_{y}
\end{array}\right\}=\sum_{k=1}^{n=8}\left[H(\bar{\omega})_{i j}\right]\left\{\int_{z_{k-1}}^{z_{k}}\left\{\begin{array}{l}
\gamma_{x z}^{\prime} \\
\gamma_{y z}^{\prime}
\end{array}\right\} d z\right\} \quad \ldots i, j=4,5
\end{aligned}
$$

Thus in matrix form

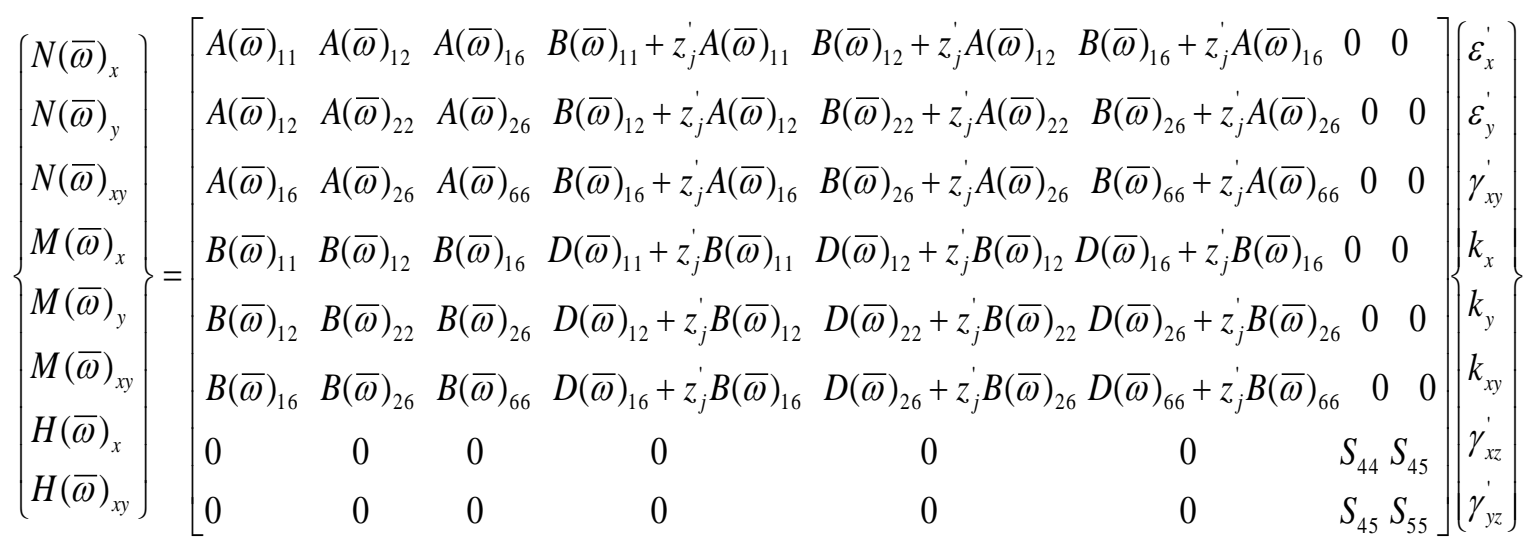

where $[A(\bar{\omega})]$ denotes the extension coefficients, $[B(\bar{\omega})]$ denotes the bending-extension coupling coefficient and $[D(\bar{\omega})]$ denotes the bending stiffness coefficients. For the element 1 the relationship among these coefficients can be given as

$$
\left(\left[A(\bar{\omega}),\left[B(\bar{\omega}),[D(\bar{\omega})]=\int_{-\frac{h}{2}}^{z_{A}}[\bar{H}(\bar{\omega})]\left(1, z, z^{2}\right) d z+\int_{z_{A}}^{\frac{h}{2}}[\bar{H}(\bar{\omega})]\left(1, z, z^{2}\right) d z\right.\right.\right.
$$

where $[\bar{H}(\bar{\omega})]$ represents the transformed reduced stiffness [59] while $z_{t}^{o}$ denotes the z-co-ordinate of midplane of $t$-th sub-laminate. The formulation of the multi-point constraint conditions leads to un-symmetric stiffness matrix. The resultant forces, $\{N(\bar{\omega})]$, moments, $\{M(\bar{\omega})]$ and transvers shear resultants, $\{H(\bar{\omega})\}$ at the delamination point satisfy the given equilibrium conditions,

$$
\begin{gathered}
\{N(\bar{\omega})]=\{N(\bar{\omega})\}_{1}=\{N(\bar{\omega})\}_{2}+\{N(\bar{\omega})]_{3} \\
\{M(\bar{\omega})]=\{M(\bar{\omega})\}_{1}=\{M(\bar{\omega})\}_{2}+\{M(\bar{\omega})]_{3}+z_{2}^{\prime}\{N(\bar{\omega})]_{2}+z_{3}^{\prime}\{N(\bar{\omega})\}_{3} \\
\{H(\bar{\omega})]=\{H(\bar{\omega})\}_{1}=\{H(\bar{\omega})\}_{2}+\{H(\bar{\omega})]_{3}
\end{gathered}
$$




\section{Hybrid HDMR based FE algorithm for layer-wise stochastic modelling of delaminated composites}

Stochastic dynamic analysis of delaminated composites is carried out using a hybrid HDMR based FE algorithm. Detail mathematical formulation for the HDMR based surrogate model is provided as APPENDIX. This article focuses on the coupled effect of source-uncertainty (due to inherent randomness in material and geometric parameters) and delamination with different degree of severity and location. The effect of noise is investigated in the surrogate based uncertainty quantification algorithm. In this paper, the combined effect of geometric and material uncertainties along with delamination in laminated composites is considered as follows:

$$
\Im(\bar{\omega})=g\left\{E_{1}(\bar{\omega}), E_{2}(\bar{\omega}), G_{12}(\bar{\omega}), G_{13}(\bar{\omega}), G_{23}(\bar{\omega}), \mu(\bar{\omega}), \rho(\bar{\omega}), \theta(\bar{\omega})\right\}+\aleph(\psi, \bar{\lambda})+\varsigma(s)
$$

where $\aleph(\psi, \lambda)$ represents the effect of delamination. The parameters $\psi$ and $\lambda$ are used to denote the location and severity of delamination. The quantity $\varsigma(s)$ represents the effect of noise in the surrogate based analysis, wherein $\mathrm{s}$ is used to denote the noise level. The compound effect of materials and geometric sourceuncertainties can be expressed as

$$
\begin{aligned}
& g\left\{E_{1}(\bar{\omega}), E_{2}(\bar{\omega}), G_{12}(\bar{\omega}), G_{13}(\bar{\omega}), G_{23}(\bar{\omega}), \mu(\bar{\omega}), \rho(\bar{\omega}), \theta(\bar{\omega})\right\} \\
& \quad=\left\{\begin{array}{l}
\phi^{1}\left(E_{1(1)} \ldots E_{1(l)}\right), \phi^{2}\left(E_{2(1)} \ldots E_{2(l)}\right), \phi^{3}\left(G_{12(1)} \ldots G_{12(l)}\right), \phi^{4}\left(G_{13(1)} \ldots G_{13(l)}\right), \\
\phi^{5}\left(G_{23(1)} \ldots G_{23(l)}\right), \phi^{6}\left(\mu_{(1)} \ldots \mu_{(l)}\right), \phi^{7}\left(\rho_{(1)} \ldots \rho_{(l)}\right), \phi^{8}\left(\theta_{(1)} \ldots \theta_{(l)}\right)
\end{array}\right\}
\end{aligned}
$$

where $\theta_{\mathrm{i}}$ denotes the ply-orientation angle, $\rho_{\mathrm{i}}$ is the mass density, $\mathrm{G}_{12(\mathrm{i})}$ and $\mathrm{G}_{23(\mathrm{i})}$ represent the shear moduli, $E_{1(\mathrm{i})}$ and $E_{2(\mathrm{i})}$ are Young's modulus along longitudinal direction and transverse direction, while ' $l$ ' represents the number of layer in the laminated composite plate. The parameter $\bar{\omega}$ is the stochasticity in input parameters. It is considered that the randomness in input parameters is distributed within a certain band of tolerance from central deterministic mean value which follows a uniform distribution. For presenting numerical results, it is considered as $10 \%$ and $\pm 10^{\circ}$ for material properties and ply orientation angle respectively according to industry standard, unless otherwise indicated. Figure 3 shows the hybrid surrogate (HDMR) based FE algorithm for uncertainty quantification followed in this paper. A description about Monte Carlo simulation can be found in Naskar et al. (2018) [57].

Besides the source-uncertainties in material and geometric properties $(g(\bar{\omega}))$, another source of uncertainty is considered in the present analysis in terms of noise $(\varsigma(s))$. The simulated noise is introduced to account for the effect of measurement error of responses, modeling and simulation error and other epistemic 


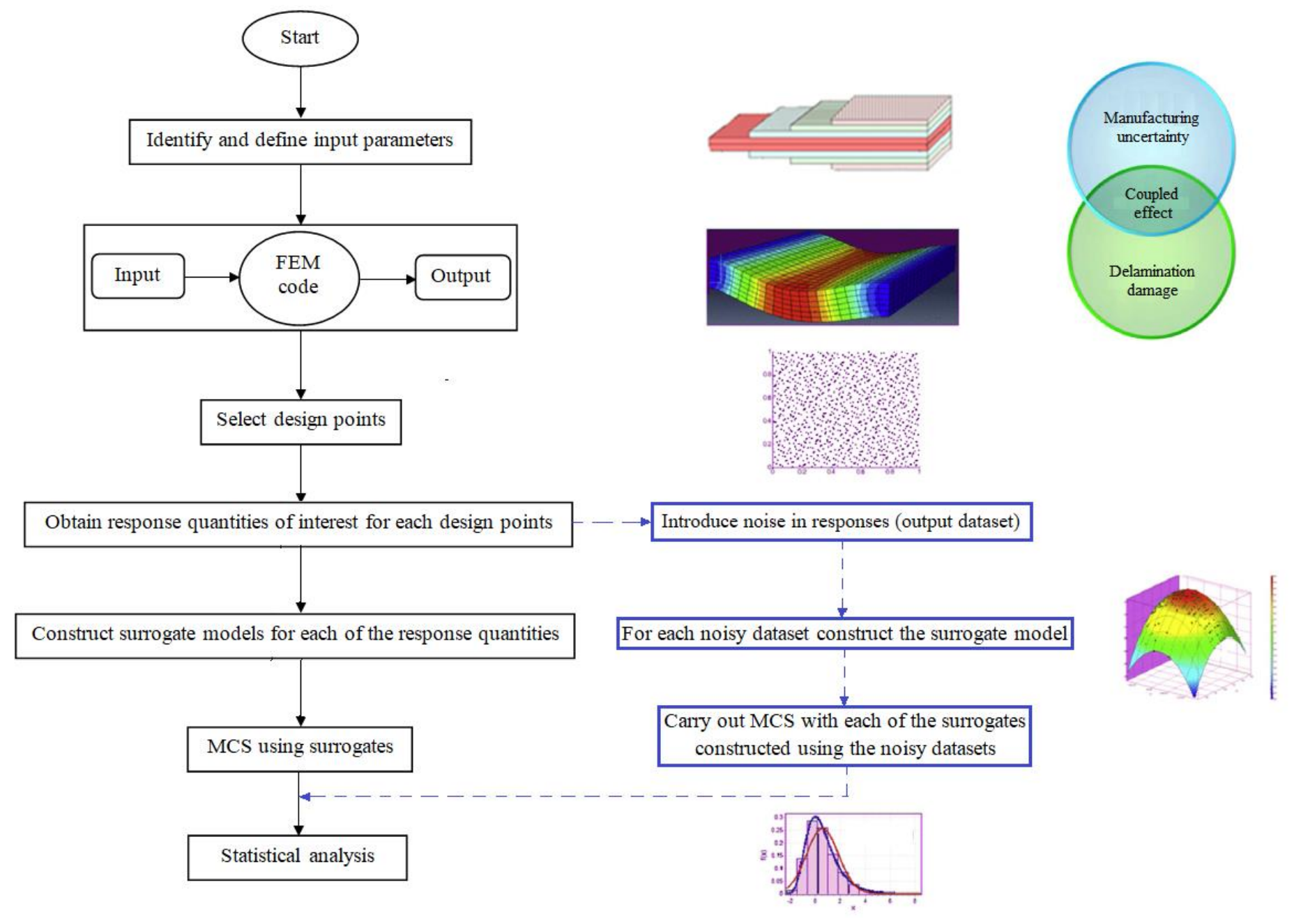

Fig. 3 Flowchart for surrogate based uncertainty quantification of composite laminates (to analyze the coupled effect of source-uncertainty and delamination) including the influence of noise. Representative figures of finite element analysis, sobol's quasi-random sampling, surrogate modelling and probability distributions are shown corresponding to the respective steps.

uncertainties involved in the system [59]. While formation of the surrogate model, gaussian white noise with a specific level $(s)$ is induced in the set of output responses as

$$
f_{i j N}=f_{i j}+s \times \xi_{i j}
$$

where, $f$ represents the natural frequency corresponding to a particular mode of vibration, subscript $i$ is the frequency number, and subscript $j$ is sample number in the design point set. The parameter $\xi_{i j}$ denotes a function that creates random numbers. Here the subscript $N$ is used to represent the noisy frequency. Thus the simulated noisy dataset is generated by considering a gaussian noise in the responses, while the input design points remain constant. Thereby Monte Carlo Simulation is carried out for each noisy dataset following a nonintrusive method as shown in Figure 3. Thus thousands of surrogate based Monte Carlo Simulations are performed with the noisy design points to quantify the effect of noise corresponding to a particular level. 


\section{Results and discussion}

In the present study, the first three natural frequencies of a cantilever laminated composite plate with length $1 \mathrm{~m}$, breadth $1 \mathrm{~m}$ and thickness $0.0004 \mathrm{~m}$ are analysed corresponding to different ply orientation angle, degree of orthotropy, degree of stochasticity, material and geometric properties, position of delamination, and number of delamination. Both deterministic as well as stochastic results are presented for a comprehensive analysis. The material properties of the composite plate are assumed as: $E_{1}=138.0 \mathrm{GPa}, E_{2}=8.96 \mathrm{GPa}$ and $\mu$ $=0.3$, while values of $G_{12}, G_{13}$, and $G_{23}$ are determined by relationship of $E$ and $\mu$ as $G_{12}=G_{13}=7.1 \mathrm{GPa}, G_{23}$ $=2.84 \mathrm{GPa}$, where the symbols have usual meanings. The first three natural frequencies are abbreviated as FNF (first natural frequency), SNF (second natural frequency) and TNF (third natural frequency) respectively.

\subsection{Validation and convergence study}

In the surrogate assisted stochastic analysis of laminated composites, two different forms of validation and convergence study are needed to be carried out. The first validation is for the finite element model of delaminated composite plate along with mesh convergence study. A second type of validation is also needed here concerning the performance (efficiency and accuracy) of the surrogate model in predicting the responses along with a convergence study for minimizing the number of design points required for forming surrogate models. Table 1 shows the mesh convergence study and comparative results with scientific literature for the deterministic first natural frequency of an undelaminated composite plate. Keeping the computational aspect in mind, a $6 \times 6$ mesh is adopted in this work. Further the deterministic first natural frequency is validated with Krawczuk et al. [72] for delaminated composite plates considering various relative position of delamination as shown in figure 4.

The optimum number of samples (drawn from Sobol sequence) to construct surrogate models are decided based on sample-wise prediction performance (scatter plot) and a comparative assessment with direct Monte Carlo simulation (probability density function plot). Based on the results presented in figure 5, a sample size of 1024 is adopted for surrogate model formation. A good agreement between the probabilistic descriptions of natural frequencies and minimal deviation from the diagonal lines of the scatter plots corroborate the accurate prediction capability of the surrogate models corresponding to the chosen sample size. It can be noted in this context that the computational time required is exorbitently high for evaluating the probabilistic responses through full scale direct MCS because of the involvement of large number of finite element simulations $\left(\sim 10^{4}\right)$. However, in case of the present surroagate based method, although a same sample 
Table 1 Non-dimensional fundamental natural frequencies $\left[\omega=\omega_{\mathrm{n}} \mathrm{L}^{2} \sqrt{ }\left(\rho / \mathrm{E}_{1} \mathrm{~h}^{2}\right)\right]$ of three layered $[\theta,-\theta, \theta]$ graphite- epoxy twisted plates, $\mathrm{L} / \mathrm{b}=1, \mathrm{~b} / \mathrm{h}=20, \psi=30^{\circ}$

\begin{tabular}{c|c|c|c|c|c}
\hline \multirow{2}{*}{$\begin{array}{c}\text { Ply orientation } \\
\text { Angle, } \theta\end{array}$} & \multicolumn{4}{|c|}{ Present FE model } & $\begin{array}{c}\text { Qatu and } \\
\text { Leissa [73] }\end{array}$ \\
\cline { 2 - 5 } & $4 \times 4$ & $6 \times 6$ & $8 \times 8$ & $10 \times 10$ & \\
\hline $15^{\circ}$ & 0.8588 & 0.8618 & 0.8591 & 0.8543 & 0.8759 \\
$30^{\circ}$ & 0.6753 & 0.6790 & 0.6752 & 0.6722 & 0.6923 \\
$45^{\circ}$ & 0.4691 & 0.4732 & 0.4698 & 0.4578 & 0.4831 \\
$60^{\circ}$ & 0.3189 & 0.3234 & 0.3194 & 0.3114 & 0.3283 \\
\hline
\end{tabular}

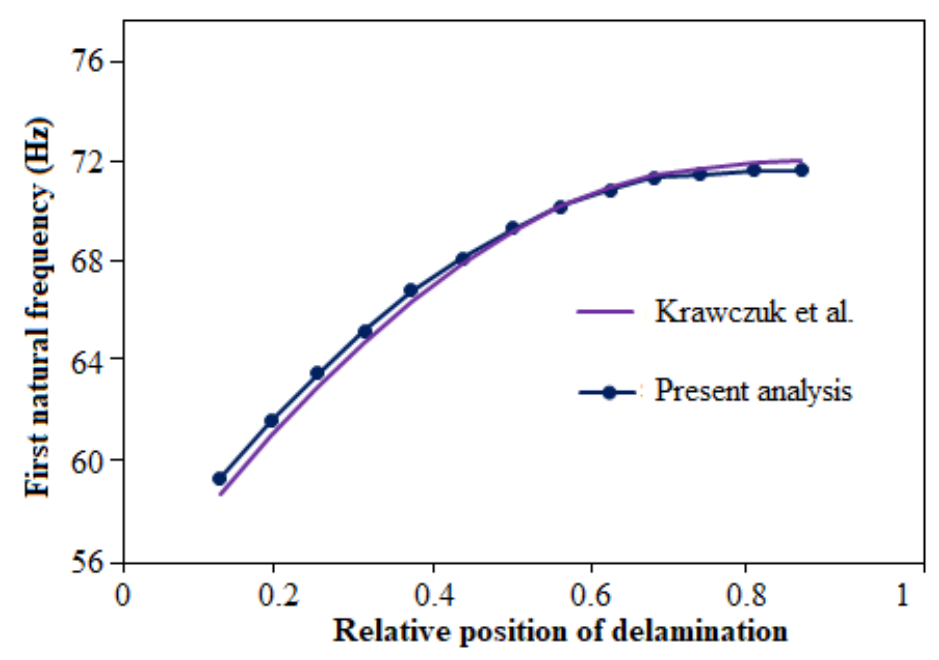

Fig. 4 Validation for the deterministic finite element code with respect to published results (Krawczuk et al. [72]) considering the effect of relative position of delamination

size as the direct MCS is considered, the requirement of carrying out actual finite element simulations is much lesser compared to the direct MCS approach. Here it is equal to the number of samples required to form the HDMR based surrogate model (i.e. 1024). Hence, the computational intensiveness (time and effort) in terms of FE analyses are decreased significantly in comparison to full-scale direct MCS.

\subsection{Deterministic analysis}

In this section, deterministic results are presented to portray the fundamental influences of the location and severity of delamination in cantilever composite plates. Table 2 presents the effect of severity of damage considering a case of single delamination. It can be noticed that the natural frequencies reduce with increasing percentage of delamination due to the reduction in stiffness. Table 3 shows the effect of single delamination in a composite plate considering different locations of delamination along the span ( $x-y$ plane). From the table, it can be noted that all three natural frequencies decrease with changing the delamination location from near fixed end to near the free end. The effect of locational variation of delamination across the thickness is shown in figure 6, which reveals an interesting trend of reduction of the frequencies up to mid-zone of the 


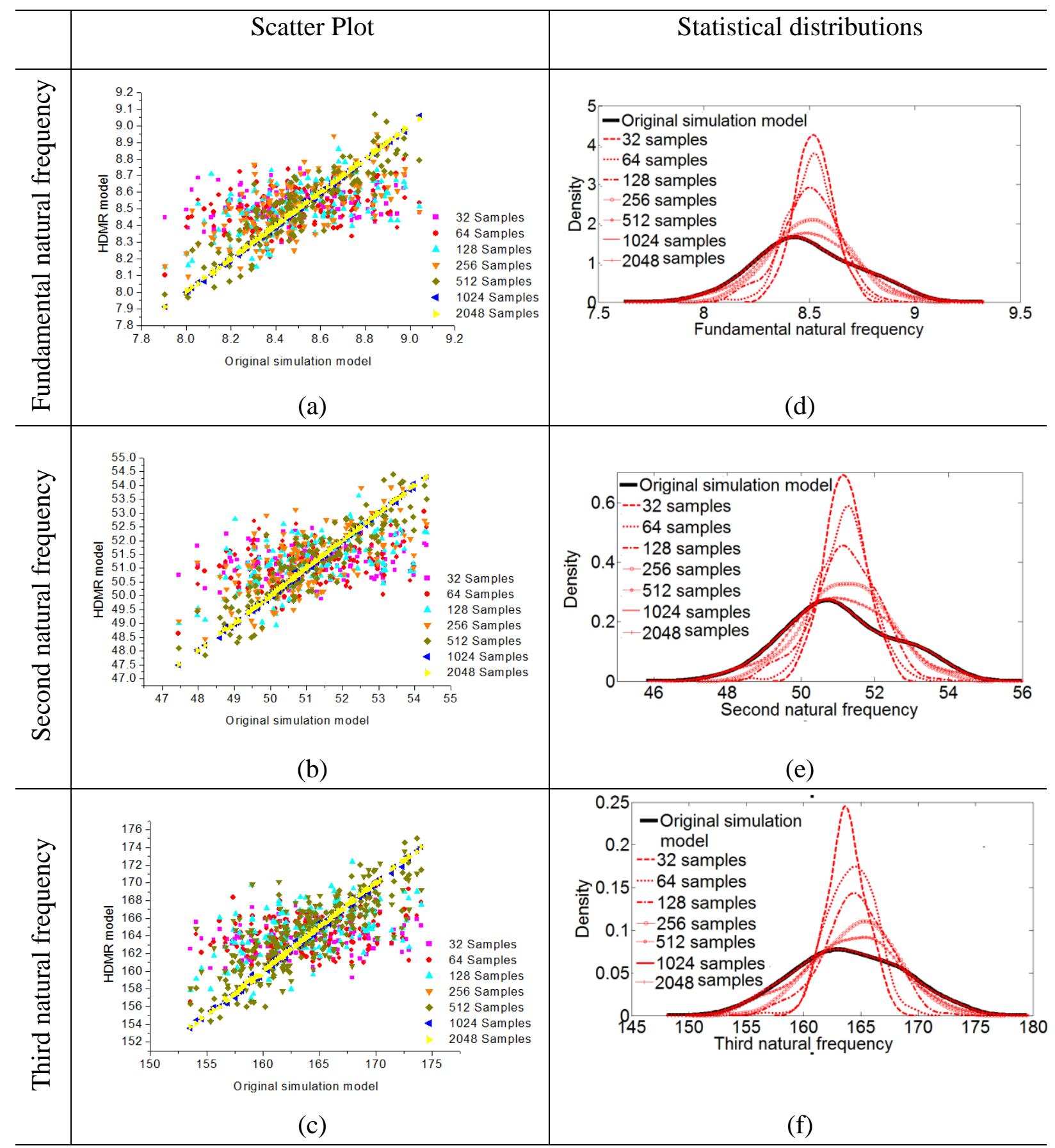

Fig. 5 (a,b,c) Scatter plots and (d, e, f) probability density function (PDF) plots for first three natural frequencies $(\mathrm{rad} / \mathrm{s})$ considering HDMR model with respect to original finite simulation model considering different sample size

interfaces and then a rise following a symmetric pattern. In this figure the results are presented considering a higher number of plies to portray the effect of locational variation of delamination across thickness more clearly. For the case of single delamination, it can be noted that the effect of increasing severity of damage (percentage of delamination) is rather less, although the natural frequencies decrease marginally with the increase of damage level. In contrast, the effect of multiple delamination is observed to be more noteworthy for the natural frequencies as shown in Table 4 considering different laminate configurations. With the increase in number of delamination $\left(n_{d}\right)$, the natural frequencies are found to decrease significantly. 
Table 2 The effect of severity of delamination ( $\%$ of delamination) on first three deterministic natural frequencies considering a stacking sequence of $\left[45^{\circ} /-45^{\circ} / 45^{\circ}\right]$

\begin{tabular}{c|c|c|c|c}
\hline \multicolumn{2}{c|}{ Parameters } & FNF & SNF & TNF \\
\hline \multicolumn{2}{c|}{ Undelaminated composite plate } & 8.851 & 51.053 & 164.702 \\
\hline \multirow{3}{*}{$\%$ of delamination } & $16.66 \%$ & 8.836266 & 51.02038 & 164.5625 \\
\cline { 2 - 5 } (Mid-point delamination) & $25 \%$ & 8.835081 & 51.01272 & 164.5404 \\
\cline { 2 - 5 } & $33.33 \%$ & 8.830061 & 50.99974 & 164.4979 \\
\cline { 2 - 5 } & $50 \%$ & 8.738607 & 50.81708 & 163.891 \\
\hline
\end{tabular}

Table 3 The effect of location of delamination (along the span) on first three deterministic natural frequencies considering a stacking sequence of $\left[45^{\circ} /-45^{\circ} / 45^{\circ}\right]$

\begin{tabular}{c|c|c|c|c}
\hline \multicolumn{2}{c|}{ Parameters } & FNF & SNF & TNF \\
\hline \multicolumn{2}{c|}{ Undelaminated composite plate } & 8.851 & 51.053 & 164.702 \\
\hline $\begin{array}{c}\text { Location of delamination } \\
\text { (Along span - 25\% } \\
\text { delamination) }\end{array}$ & Near fixed end & 8.842589 & 51.03411 & 164.6553 \\
\cline { 2 - 5 } & Mid-point & 8.835081 & 51.01272 & 164.5404 \\
\cline { 2 - 5 } & Near Free end & 8.398455 & 50.04205 & 161.7076 \\
\hline
\end{tabular}

\subsection{Stochastic analysis}

Stochastic results are presented in this section for natural frequencies of delaminated composite plates considering the compound variation of the source-uncertainties (refer to section 2) along with the effect of noise. Figure 7-13 and figure 15 show stochastic results concerning different aspects of single delamination, while figure 14 considers multiple delamination in composites. Figure 7 shows the effect of increasing percentage of delamination (along with the case of no delamination) on the first three natural frequencies of composite laminates, wherein it can be noticed that the natural frequencies reduce marginally with the increase in severity of damage while the probabilistic descriptions vary considerably. The effect of plyorientation angle on the stochastic natural frequencies of delaminated composite plates (single delamination $25 \%$ ) is shown in figure 8 considering cross-ply and angle-ply laminates. The figure illustrates that cross-ply laminates have higher range of natural frequencies compared to angle-ply laminates, whereas the probabilistic response bounds are more in case of angle ply laminates. Figure 9 shows the effect of variation in degree of orthotropy (DOO) on the stochastic natural frequencies of delaminated composite plates (considering single delamination $-25 \%$ ), wherein all the natural frequencies along with their probabilistic bounds are found to increase with the increase in degree of orthotropy. The effect of degree of stochasticity (i.e. level of source- 


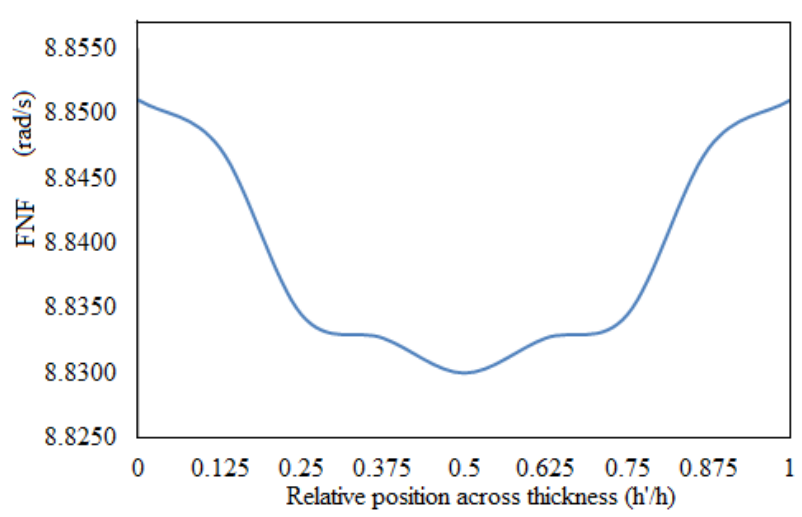

(a)

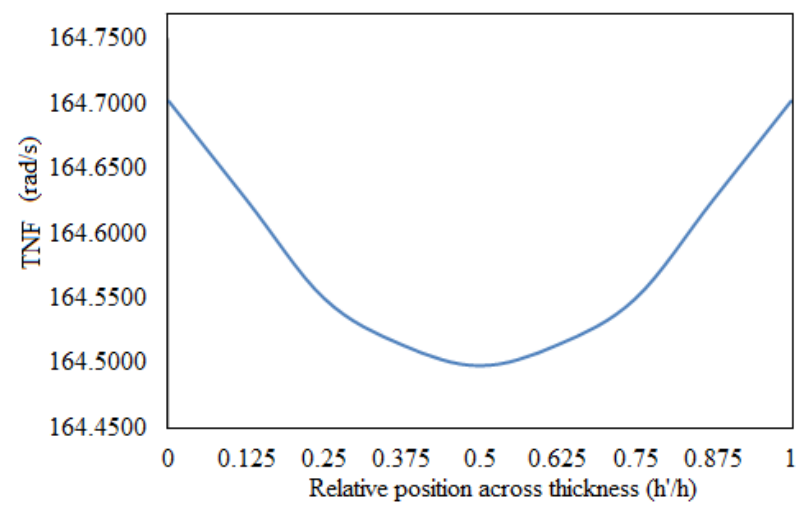

(c)

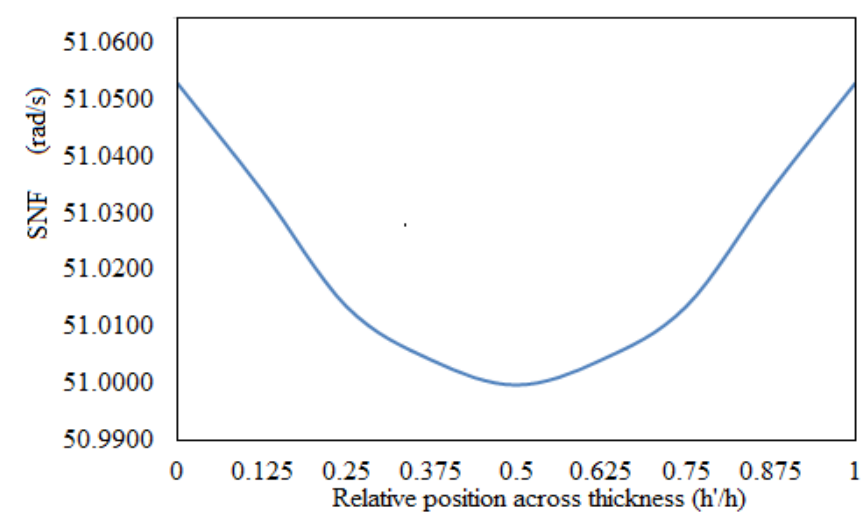

(b)

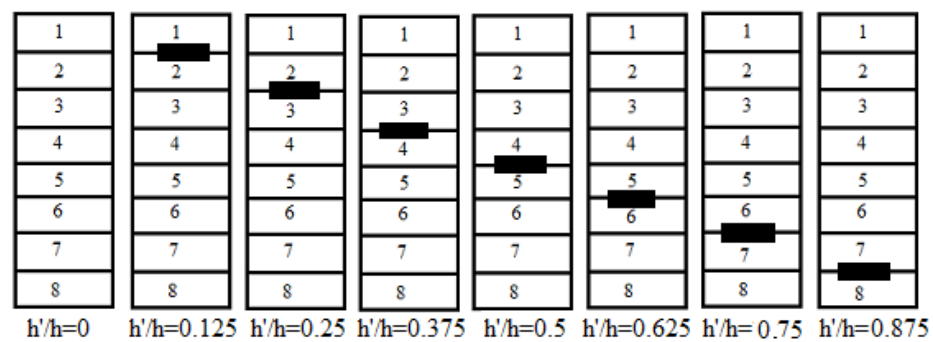

(d)

Fig. 6 (a-c) Variation of first three natural frequencies of an eight layered angle ply $\left[45^{\circ} /-45^{\circ} / 45^{\circ} /-45^{\circ}\right]_{\mathrm{S}}$ delaminated composite plate (single delamination) with varying relative location of delamination across the thickness (d) Relative location of delamination (at the interfaces of two laminas) across thickness

Table 4 Effect of multiple delamination (33.33\% mid-point delamination) on the first three natural frequencies for various laminate configurations of a $[\theta /-\theta / \theta /-\theta]_{\mathrm{S}}$ family of composite

\begin{tabular}{c|c|c|c|c|c|c|c|c|c}
\hline \multirow{2}{*}{$\begin{array}{c}\text { Ply } \\
\text { orientation } \\
\text { angle }(\theta)\end{array}$} & \multicolumn{3}{|c|}{$\begin{array}{c}\text { No delamination } \\
\left(n_{d}=0\right)\end{array}$} & \multicolumn{3}{c|}{$\begin{array}{c}\text { Single delamination } \\
\left(n_{d}=1\right)\end{array}$} & \multicolumn{3}{c}{$\begin{array}{c}\text { Multiple delamination } \\
\left(n_{d}=4\right)\end{array}$} \\
\cline { 2 - 10 } & FNF & SNF & TNF & FNF & SNF & TNF & FNF & SNF & TNF \\
\hline $0^{\circ}$ & 8.016 & 35.627 & 115.711 & 7.954 & 35.438 & 115.314 & 7.935 & 35.378 & 115.196 \\
\hline $15^{\circ}$ & 8.107 & 39.262 & 133.232 & 8.056 & 39.122 & 132.872 & 8.046 & 39.078 & 132.758 \\
\hline $30^{\circ}$ & 8.467 & 46.254 & 158.215 & 8.434 & 46.189 & 157.974 & 8.431 & 46.171 & 157.904 \\
\hline $45^{\circ}$ & 8.851 & 51.053 & 164.702 & 8.830 & 50.999 & 164.497 & 8.829 & 50.986 & 164.448 \\
\hline $60^{\circ}$ & 9.072 & 52.012 & 158.057 & 9.053 & 51.93 & 157.749 & 9.051 & 51.919 & 157.664 \\
\hline $75^{\circ}$ & 8.951 & 50.296 & 145.491 & 8.931 & 50.236 & 145.167 & 8.927 & 50.220 & 145.074 \\
\hline $90^{\circ}$ & 8.731 & 48.202 & 134.891 & 8.710 & 48.148 & 134.559 & 8.704 & 48.133 & 134.463 \\
\hline
\end{tabular}




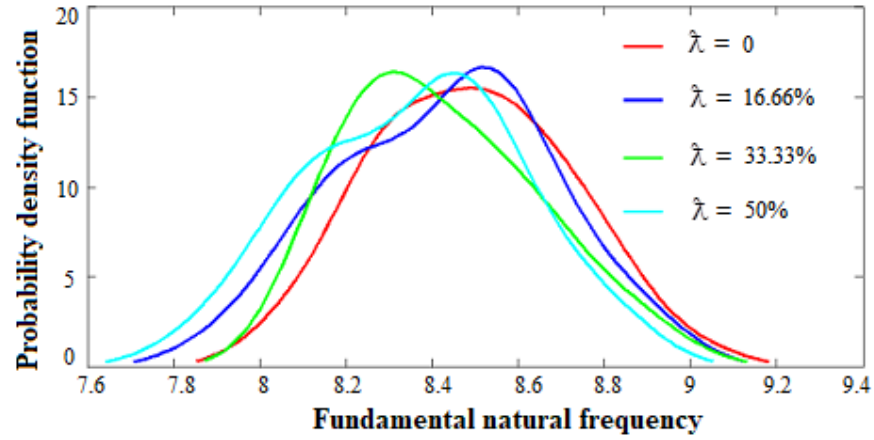

(a)

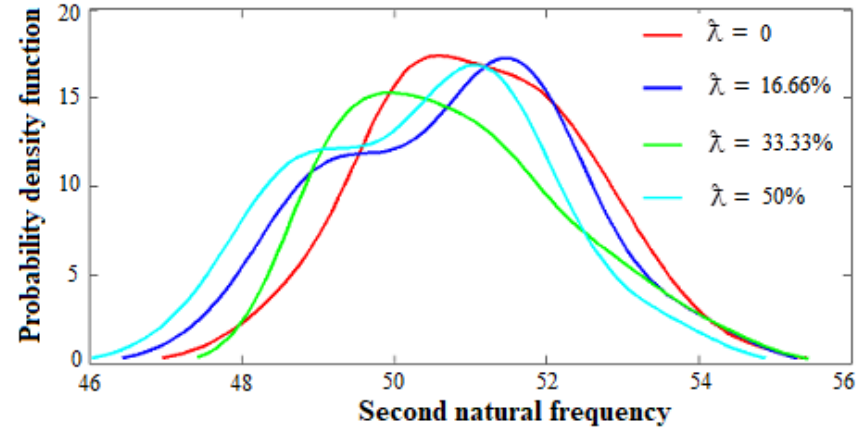

(b)

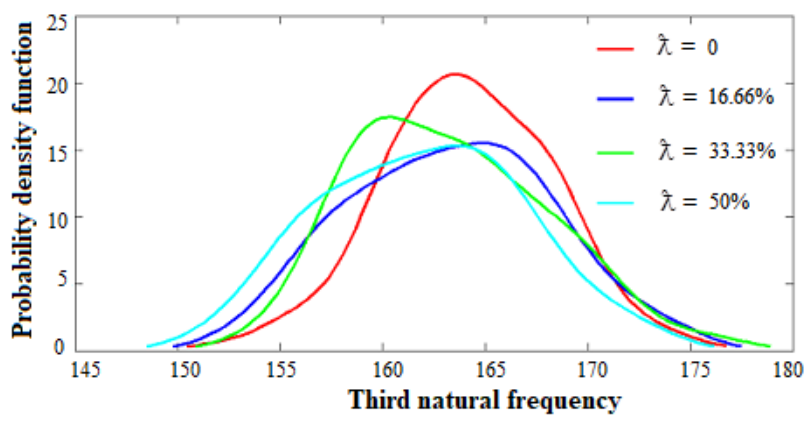

(c)

Fig. 7 Probabilistic description of first three natural frequencies ( $\mathrm{rad} / \mathrm{s}$ ) due to varying severity of delamination $(\lambda)$ considering the case of single delamination

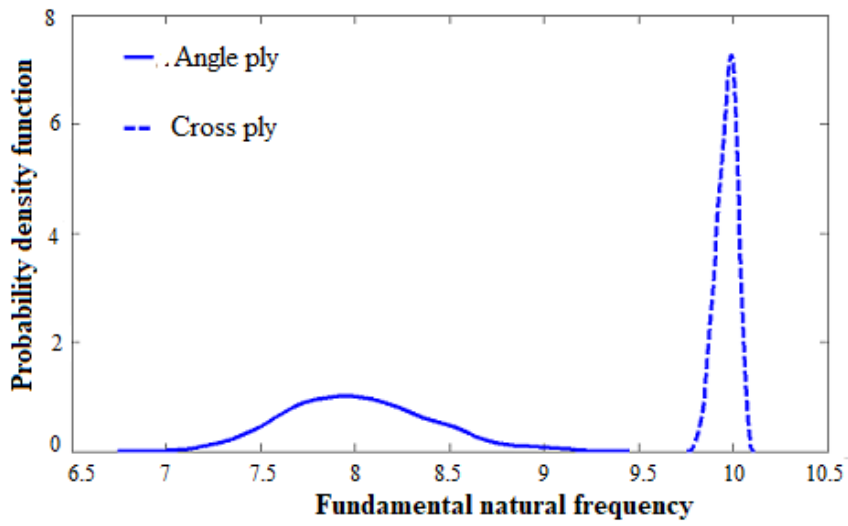

(a)

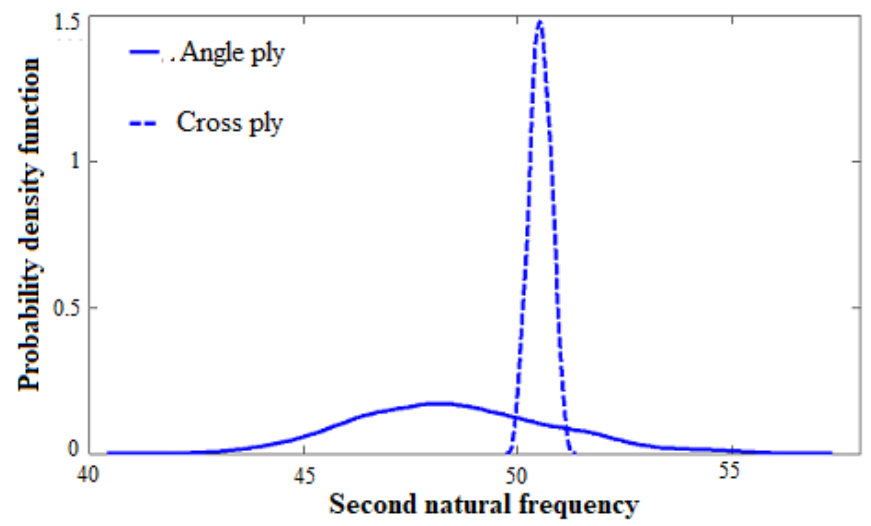

(b)

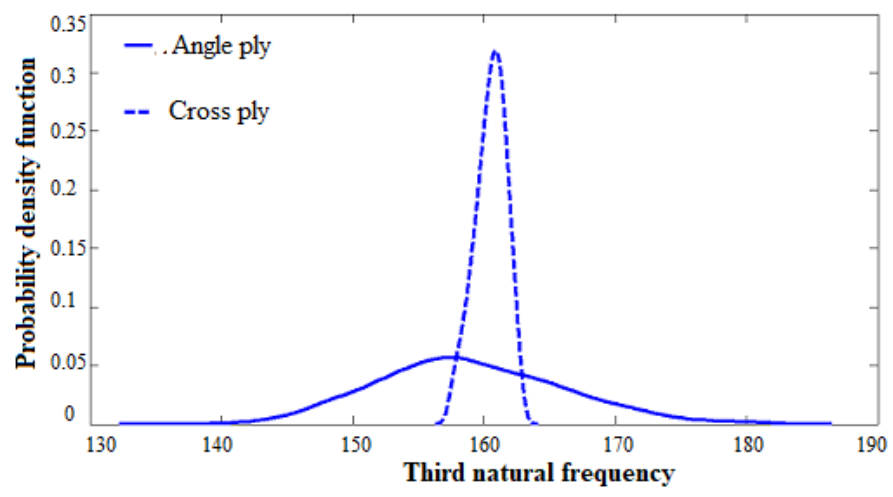

(c)

Fig. 8 Effects of delamination on first three natural frequencies ( $\mathrm{rad} / \mathrm{s}$ ) considering angle ply and cross ply laminate configurations 


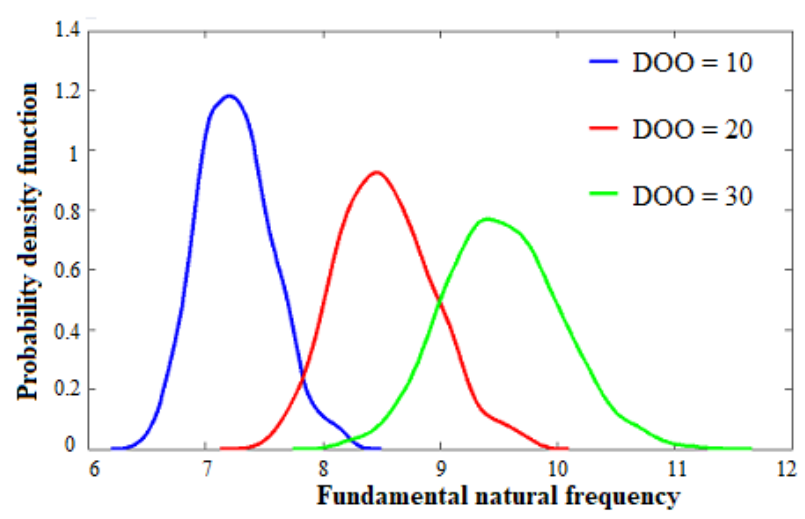

(a)

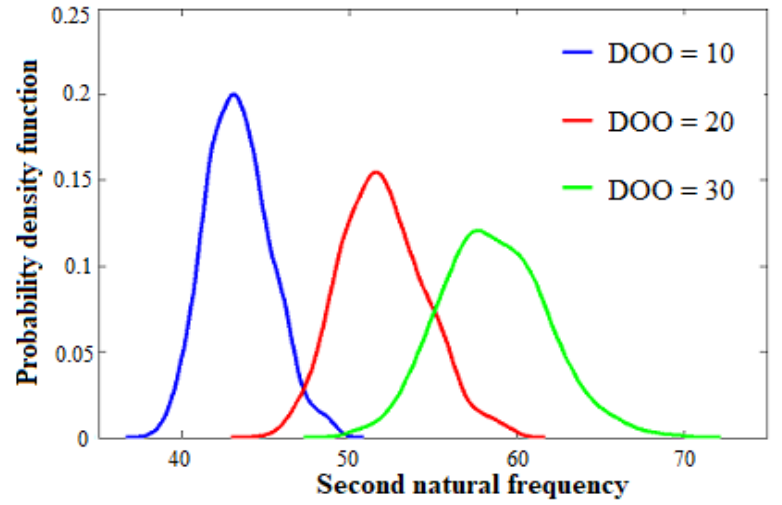

(b)

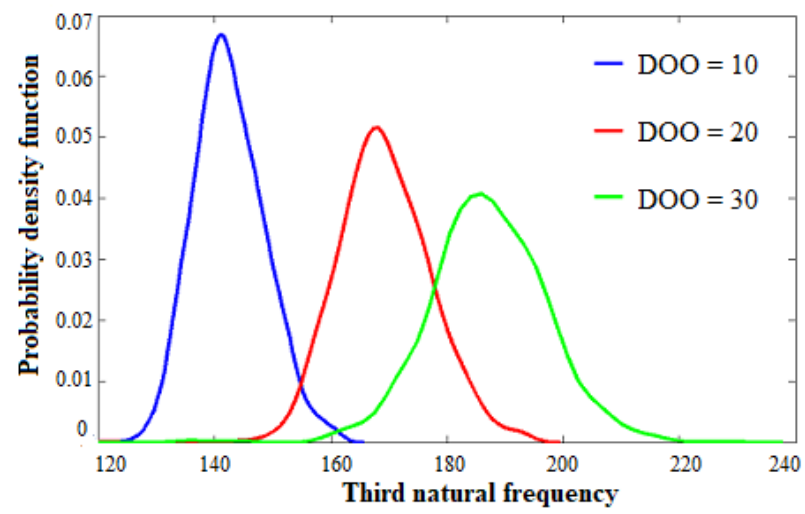

(c)

Fig. 9 Effect of variation of degree of orthotropy (DOO) on the first three natural frequencies $(\mathrm{rad} / \mathrm{s})$ of delaminated composite plates

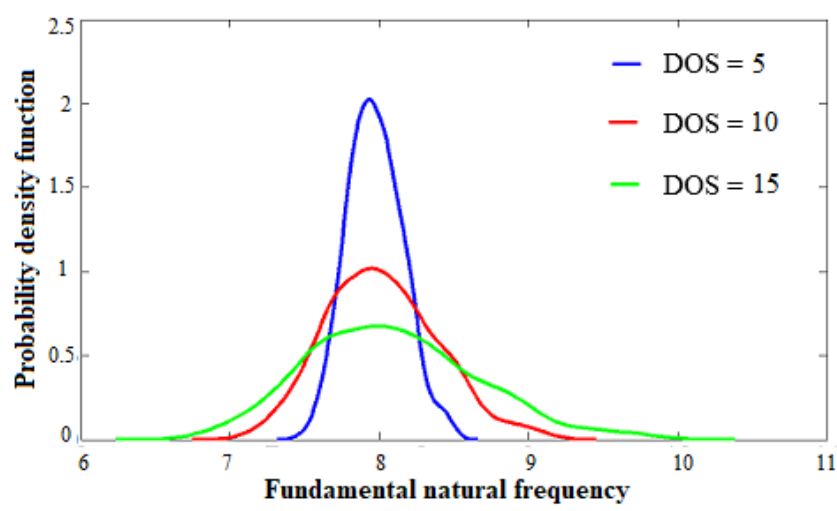

(a)

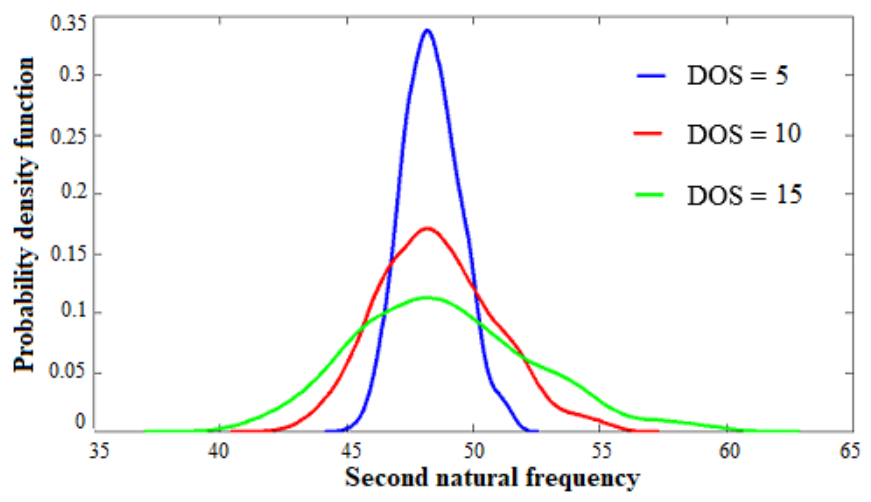

(b)

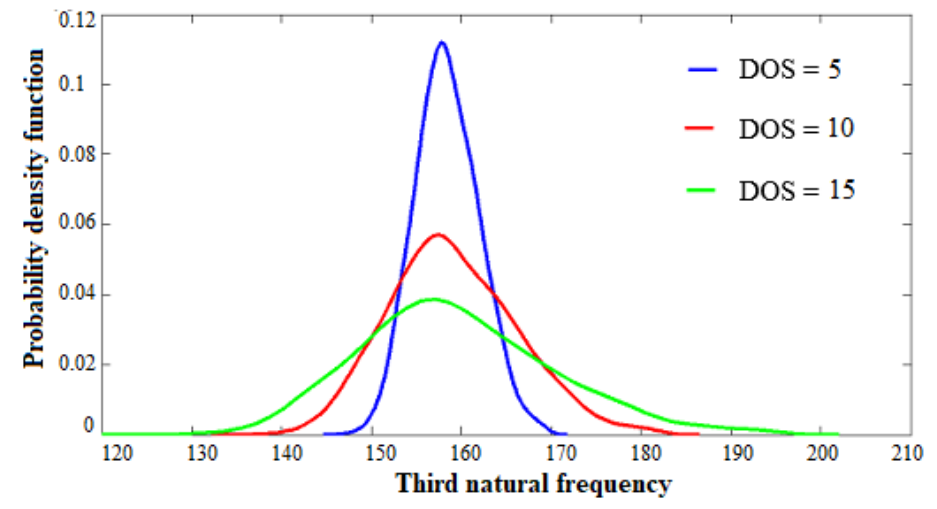

(c)

Fig. 10 Effect of variation in degree of stochasticity (DOS) on the first three natural frequencies $(\mathrm{rad} / \mathrm{s})$ of delaminated composite plates 


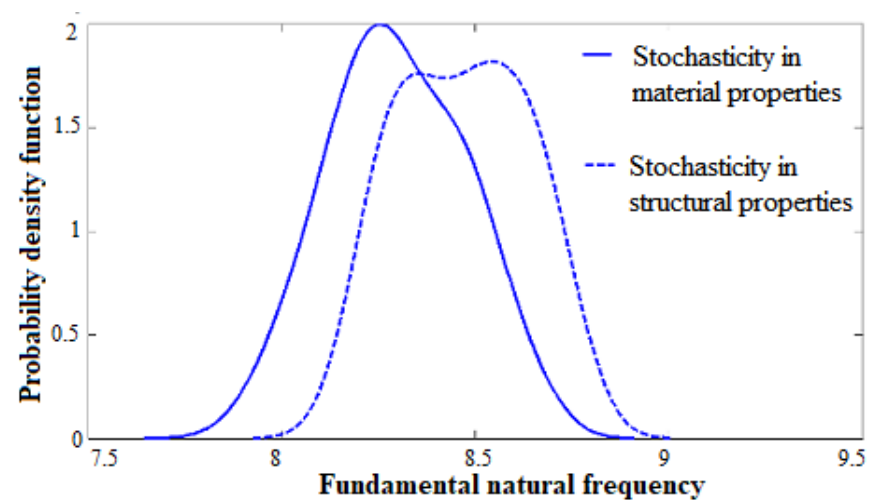

(a)

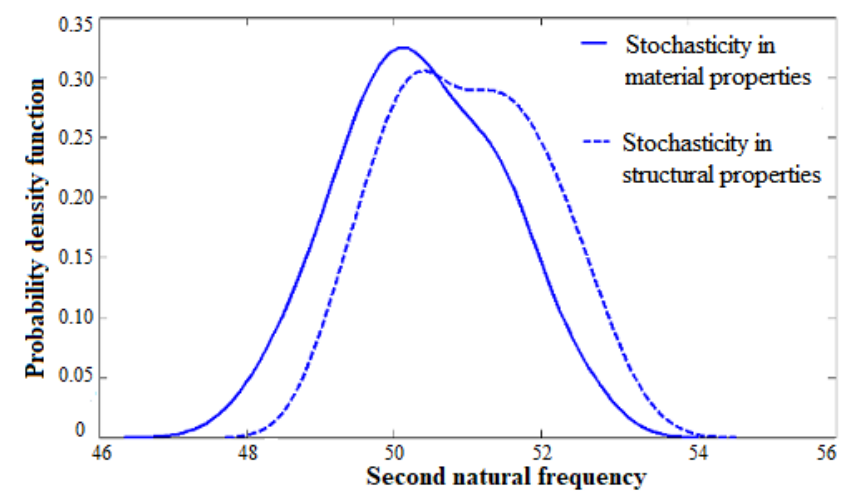

(b)

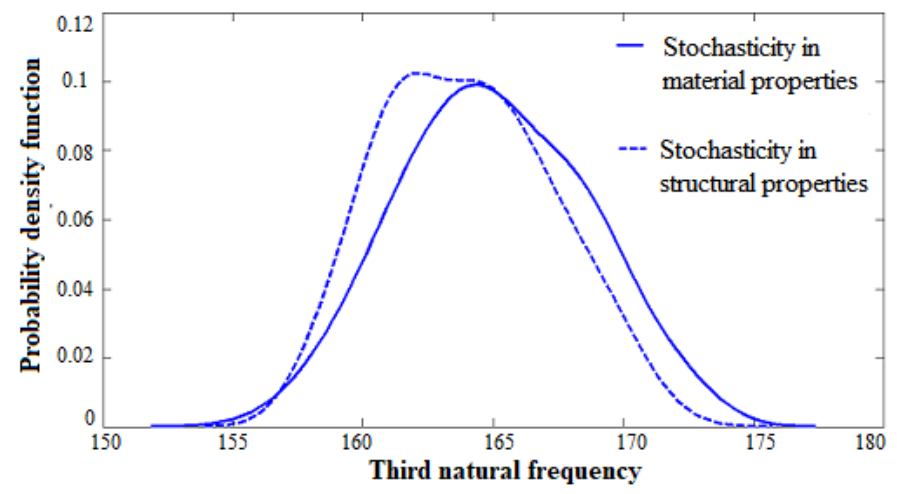

(c)

Fig. 11 Effects of individual variation of all material properties and structural attributes $(\theta)$ on the first three natural frequencies $(\mathrm{rad} / \mathrm{s})$

uncertainty) on first three natural frequencies is presented in figure 10. As expected, the response bounds are noticed to be increased with increasing degree of stochasticity. Figure 11 presents a comparative assessment in two different cases of source uncertainty in delaminated composite plates (considering single delamination $-25 \%$ ), compound effect of stochasticity in all materials properties only and the effect of stochsticity in structural property (ply orientation angle). Besides significant difference in the probability distribution between the two cases, the natural frequencies are found to decrease marginally in case of the compound variation of material properties. From the probabilistic response bounds it can be discerned that the sensitivity of ply orientation angle is one of the most predominant in the free vibration responses of composite plates.

Figure 12 and 13 show the effect of the location of delamination (considering single delamination $25 \%$ ) on stochastic natural frequencies of composite plates. Figure 12 shows the effect of span-wise location, wherein it can be noticed that the influence of delamination becomes more severe as the location changes from the fixed end to the free end in a cantilever composite plate along with an increase in probabilistic response bounds. Figure 13 presents the effect of location of delamination across the thickness of a composite plate (i.e. single delamination is considered at the interface of different layers) on the stochastic natural frequencies. While obtaining these results, a different laminate configuration with increased number of layers 


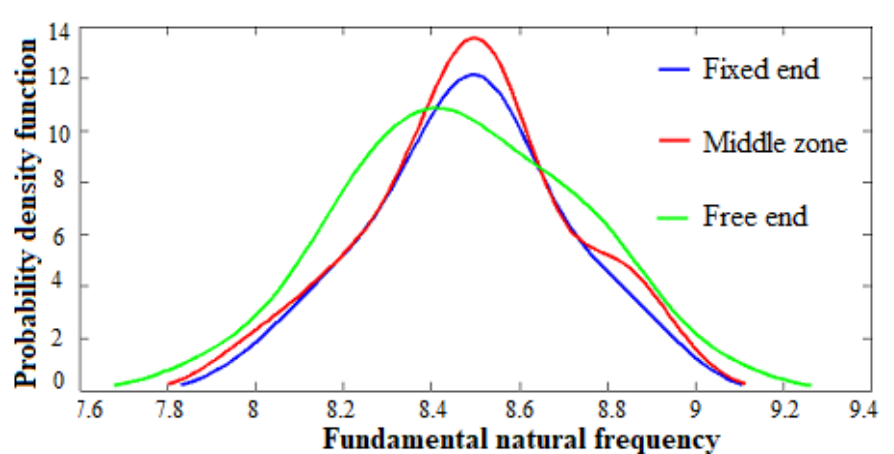

(a)

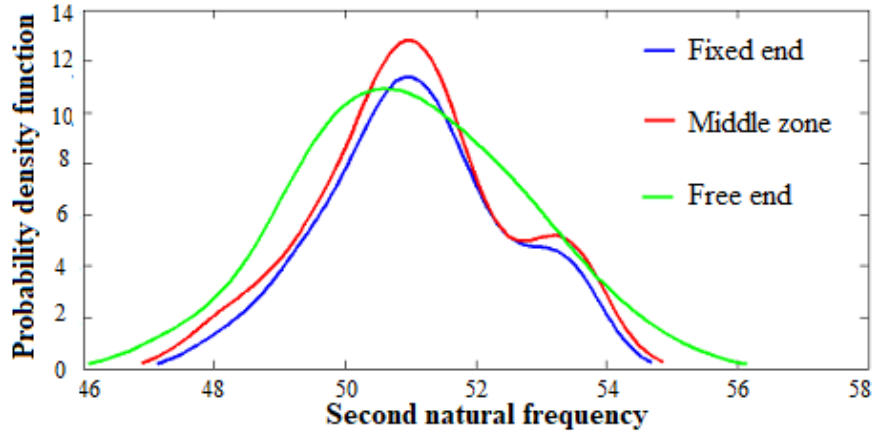

(b)

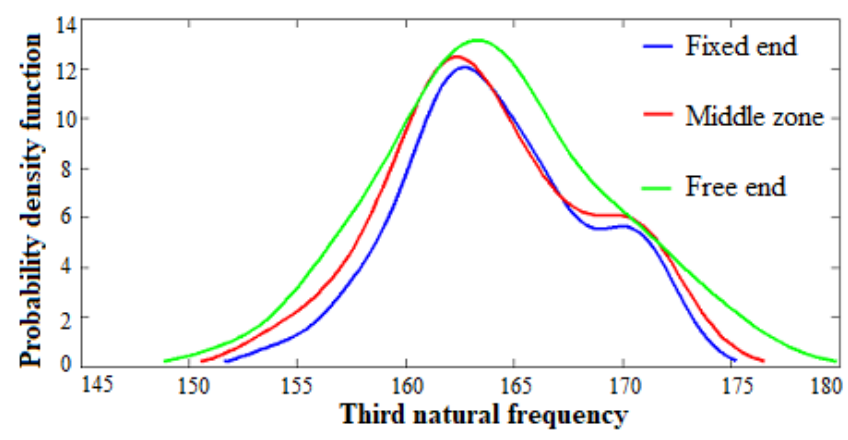

(c)

Fig. 12 Effect of delamination location on the first three natural frequencies $(\mathrm{rad} / \mathrm{s})$ considering spatial variation along the span (fixed, middle, and free end) with $25 \%$ single delamination

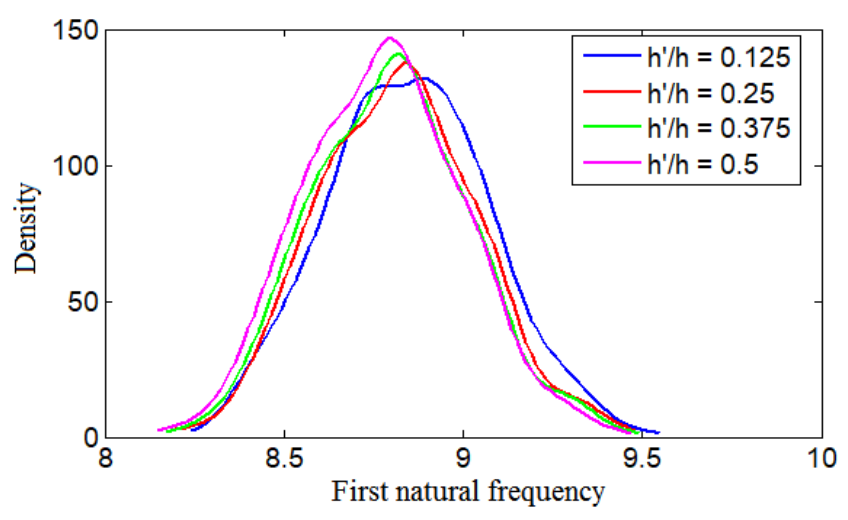

(a)

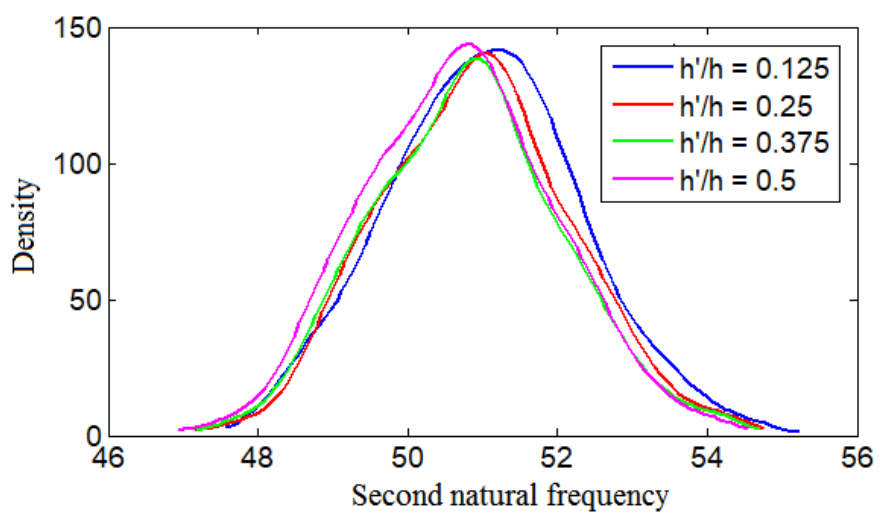

(b)

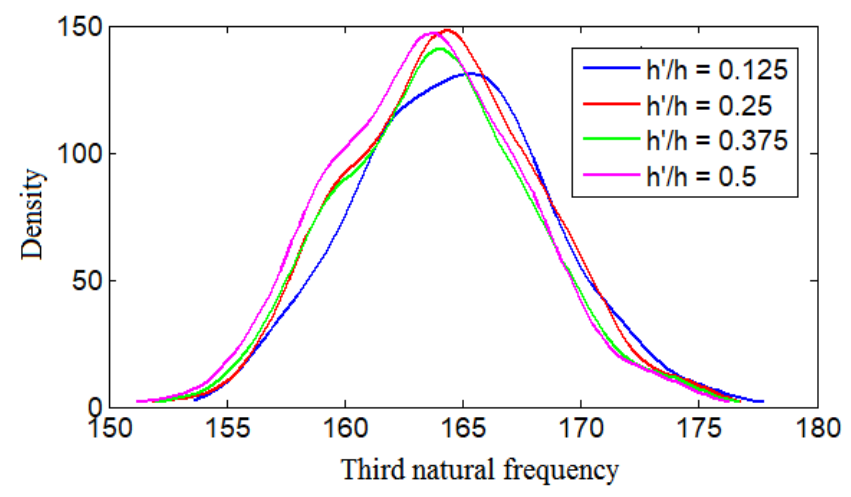

(c)

Fig. 13 Effects of delamination location across the thickness (refer to figure 6 for the pictorial representation of $\mathrm{h}^{\prime} / \mathrm{h}$ ratio) on the first three natural frequencies ( $\mathrm{rad} / \mathrm{s}$ ) considering a $25 \%$ single delamination case 


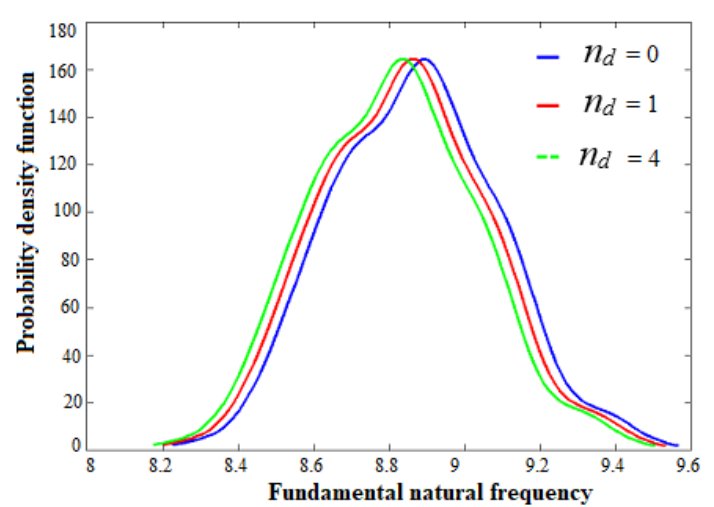

(a)

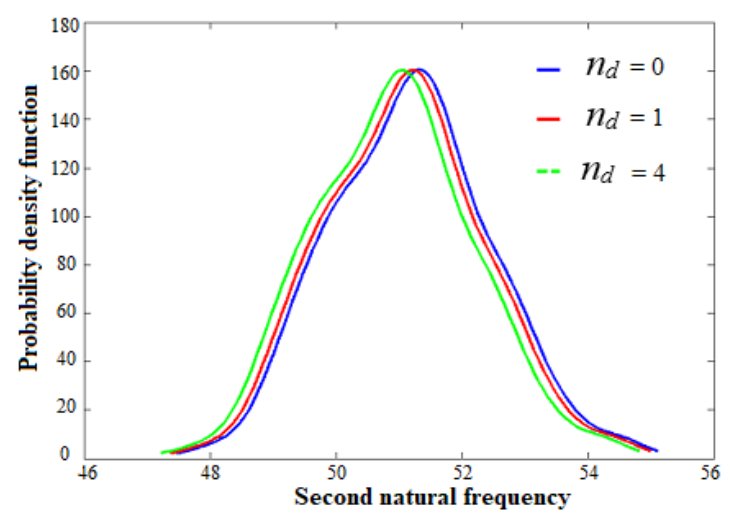

(b)

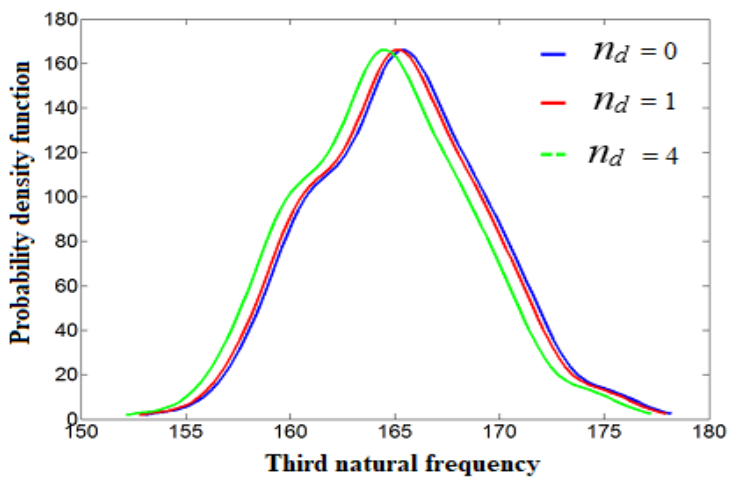

(c)

Fig. 14 Effects of number of delamination $\left(n_{d}\right)$ on the first three stochastic natural frequencies $(\mathrm{rad} / \mathrm{s})$ for the case of multiple delamination in composite plates

is used to portray the effect of the location of delamination across the thickness clearly. From the figure it can be observed that the range of natural frequencies reduce as the location of delamination varies from the two free surfaces towards the middle of thickness of the laminate.

The results in figure 14 are presented to analyse the effect of multiple delamination on stochastic natural frequencies of composite plates considering a laminate configuration of $\left(45^{\circ} /-45^{\circ} / 45^{\circ} /-45^{\circ}\right)_{\mathrm{S}}$. Comparative probability distribution plots are presented considering the case of no delamination, single delamination and four delaminations $\left(n_{d}=0,1,4\right)$. It can be noticed from the figures that the range of natural frequencies reduce with the increase in number of delaminations. The influence of noise on the stochastic natural frequencies of delaminated composite plates is presented in figure 15 considering a single delamination ( $25 \%$ mid-point delamination). It can be noticed that the stochastic bound of the probability distributions increase with the increase in level of simulated noise $(s)$.

\section{Summary and perspective}

This paper presents an efiicient stochastic bottom-up framework for analyzing the coupled effect of delamination and source-uncertainties $(\mathfrak{I}(\bar{\omega}))$ on natural frequencies of composite plates. The simulated noise studied in this article can be considered equivalent to accounting for the effect of measurement error of 


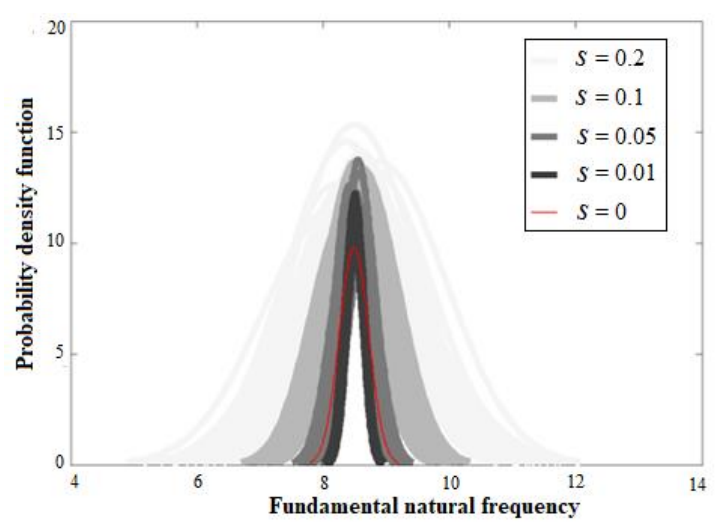

(a)

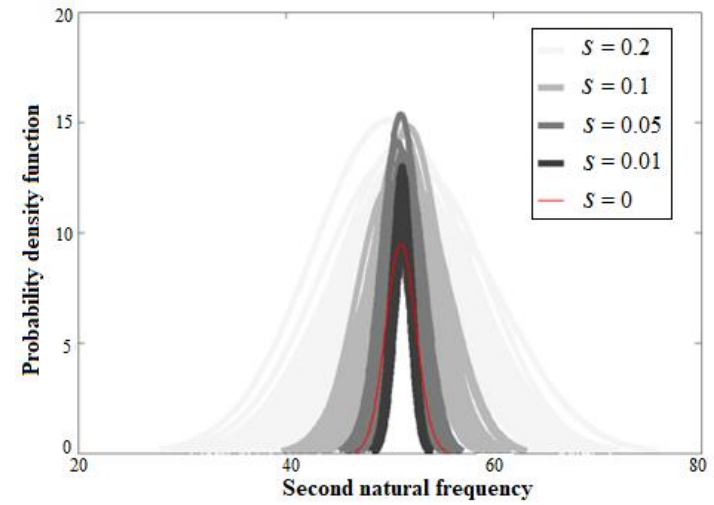

(b)

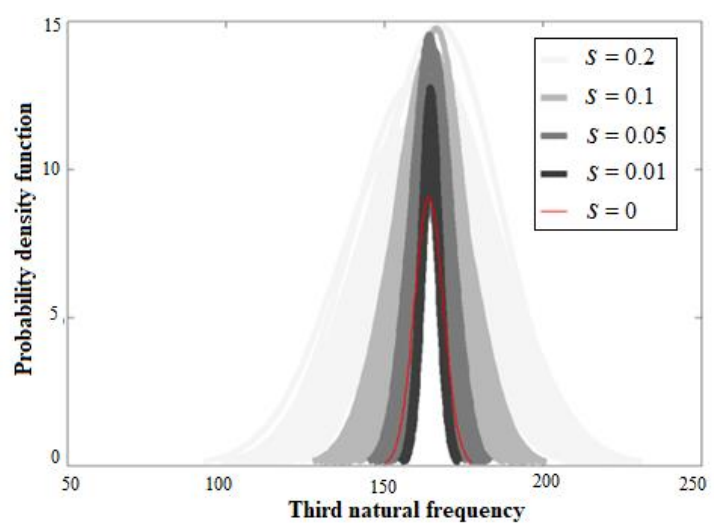

(c)

Fig. 15 Effect of noise on the first three stochastic natural frequencies ( $\mathrm{rad} / \mathrm{s})$ of delaminated composite plates responses, modeling and simulation error and other epistemic uncertainties involved in the system. A stochastic simulation involving thousands of finite element simulations $\left(\sim 10^{4}\right)$ becomes exorbitantly computationally expensive. To mitigate this lacuna, a surrogate based approach (HDMR coupled with DMORPH) in conjunction with the stochastic finite element formulation is adopted to obtain computational efficiency (without compromising the accuracy of results) in the present analysis. In case of the structural mechanics problems, where efficient analytical solutions [74-80] are not available, a surrogate based approach can be developed to carryout multiple iterative function evaluations.

In the stochastic characterization of delaminated composites, there exists three distinct stages of the analysis: uncertainty modelling at the input level, propagation of uncertainty to the global level and quantification of the global responses such as natural frequencies. A layer-wise model of source-uncertainty along with the effect of noise and delamination is adopted in the present analysis, as discussed in section 2 . Normally efficeint function evaluations (in terms of the stochastic input parameters) by direct closed-form formulae are not available for complex structural systems like laminated composites. In such situation, a numerical analysis technique like finite element method is adopted to obtain the response quantities. Finite element analyses are normally very expensive and time consuming. Inclusion of the effect of delamination in 
the present study makes the simulations more computationally intensive. The situation becomes worse in case of a stochastic analysis that requires thousands of such finite element simulations to be carried out. The surrogate based uncertainty propagation strategy, as adopted in this study, can develop a representative and predictive mathematical/ statistical metamodel relating the natural frequencies to a number of stochastic input variables. Thereafter the metamodels (response surface) are used to compute the dynamic characteristics corresponding to a given set of input variables, instead of having to simulate the time-consuming FE model repeatedly. The response surface here represents the results (or outputs) of the structural analyses encompassing (in theory) every prospective combination of the stochastic input variables. Hence, thousands of combinations of the stochastic input variables can be created and a pseudo analysis (efficient, yet accurate) for each variable set can be performed by adopting the corresponding surrogate model. The final step in the stochastic analysis is uncertainty quantification in the output responses, which is effectively carried out by deriving the probabilistic distributions and the statistical moments.

The results in this article capture the influence of inevitable source-uncertainties in material and structural attributes (manufacturing uncertainties) along with service-life conditions such as damage in composites (delamination). The effect of source-uncertainty in all material properties and structural properties are analyzed separately to ascertain their relative influence on the stochastic natural frequencies. It is noted that the ply orientation angle is more sensitive to natural frequencies compared to the individual effect of variability in material properties of the delaminated composites. Along with the previous studies [81], where the individual effect of variation in different material properties are analyzed for undelaminated composites, the present results can provide a comprehensive idea about the senstivity of various input parameters towards the global dynamic behaviour of the structure in the presence of delamination. Effect of single as well as multiple delamination on the stochastic dynamic responses of composite plates is analyzed considering the aspects of location and severity of delamination. The results reveal that the effect of increasing number of delamination is more sensitive to the natural frequencies compared to the effect of increasing severity of delamination corresponding to a particular case of single delamination. Besides the conventional sources of manufacturing uncertainty in material and structural properties, another source of uncertainty is considered in this study in the form of noise, which can be considered tantamount to incorporating various forms of errors involved in the system and other epistemic uncertainties, which are not explicitly addressed in the analysis. 


\section{Conclusion}

The compound influence of source-uncertainties in material and structural attributes (manufacturing uncertainty) and delamination (a service-life condition) on the natural frequencies of composite laminates is analyzed including the effect of inevitable noise. The propagation of source-uncertainty can cause a dramatic shift in the dynamic behavior of composites when coupled with the effect of damage. A hybrid HDMR based finite element code is developed for delaminated composite plates to study the effect of single and multiple delamination along with their locational sensitivity and severity. In-depth results are presented in both deterministic as well as stochastic regime for a comprehensive understanding. Various laminate configurations along with different degree of orthotropy and degree of stochasticity are analyzed to provide a thorough insight on the stochastic dynamic behavior of delaminated composite structures. It is found that the coupled effect of stochasticity/ source-uncertainty and delamination has significant influence on the dynamic behaviour of composite structures. Thus it is imperative to include these aspects in subsequent analyses/ design process to ensure the desired robust, safe and sustainable system performance. Future research would follow the probabilistic analysis of delaminated shell structures and consideration of other forms of damages concerning composites in the stochastic regime. The present paper dealing with the efficient hybrid HDMR based finite element analysis for the stochastic dynamics of delaminated composite plates would serve as a valuable reference in such future studies.

\section{APPENDIX}

In the case of large number of input variables (i.e. high dimensional systems), a system can be effectively analysed by using the high dimensional model representation (HDMR) [65]. By using the HDMR, deterministic as well as stochastic relationships can be handled. The HDMR is employed to create a model for prediction of the response output in the stochastic region (in this paper natural frequency is taken as output response). To confirm the component functions' orthogonality, D-MORPH algorithm is employed in the formulation. In the present approach, the function $\phi(\xi)$ is decomposed with component functions by input parameters $\xi=\left(\xi_{1}, \xi_{2}, \ldots, \xi_{k k}\right)$. The nature of the input parameters is independent and the component functions are projected by vanishing condition. So, this technique has limitation for general formulation. The output is determined for the different input variables as [65] 


$$
\begin{gathered}
\phi(\xi)=\phi_{0}+\sum_{i=1}^{k k} \phi_{i}\left(\xi_{i}\right)+\sum_{1 \leq i<j \leq k k} \phi_{i j}\left(\xi_{i}, \xi_{j}\right)+\ldots \ldots+\phi_{12 \ldots \ldots k k}\left(\xi_{1}, \xi_{2}, \ldots ., \xi_{k k}\right) \\
\phi(\xi)=\sum_{u \subseteq k k} \phi_{u}\left(\xi_{u}\right)
\end{gathered}
$$

where $\phi_{0}$ denotes the mean value, which is a zeroth order component function. $\phi_{i}\left(\xi_{i}\right)$ represents the first order component function, $\phi_{i j}\left(\xi_{i}, \xi_{j}\right)$ resents the second order component functions, and $\phi_{12 \ldots . k k}\left(\zeta_{1}, \zeta_{2}, \ldots, \zeta_{k k}\right)$ denotes the residual contribution by input parameters, while $u \subseteq\{1,2, \ldots, k k\}$ represents the subset wherein $u \subseteq k k$ for simplicity and empty set, $\eta \in u$. The correlated variables are defined as,

$$
\begin{gathered}
\left\{\phi_{u}\left(\zeta_{u} \mid u \subseteq k k\right)\right\}=A r g \min _{\left\{g_{u} \in L^{2}\left(R^{u}\right), u \subseteq k k\right\}} \int\left(\sum_{u \subseteq k} g_{u}\left(\zeta_{u}\right)-\phi(\zeta)\right)^{2} w(\zeta) d \zeta \\
\forall u \subseteq k k, \quad \forall i \in u \quad, \quad \int \phi_{u}\left(\zeta_{u}\right) w(\zeta) d \zeta_{i} d \zeta_{-u}=0 \\
\forall v \subset u, \quad \forall g_{v}: \int \phi_{u}\left(\zeta_{u}\right) g_{v}\left(\zeta_{v}\right) w(\zeta) d \zeta=\left\langle\phi_{u}\left(\zeta_{u}\right), g_{v}\left(\zeta_{v}\right)\right\rangle=0
\end{gathered}
$$

The function $\phi(\zeta)$ is determined from the sample data by experiments or by modelling. The squared error can be reduced to minimise the computational cost. Considering $Q$ in Hilbert space expanded on the basis $\left\{q_{1}, q_{2}, \ldots, q_{k k}\right\}$, the bigger subspace $\bar{Q}(\supset Q) \quad$ is expanded by extended basis $\left\{q_{1}, q_{2}, \ldots, q_{k k}, \quad q_{k k+1}, \ldots ., q_{m}\right\}$. Then $\bar{Q}$ can be decomposed as

$$
\bar{Q}=Q \oplus Q^{\perp}
$$

where $Q^{\perp}$ represents the complement subspace of $Q$ [82] within $\bar{Q}$. In the past works [83-85], basis functions are employed to determine the component functions. The basis functions $\{\varphi\}$ are used to estimate the component functions of second order HDMR expansion as [85]

$$
\begin{gathered}
\phi_{i}\left(\zeta_{i}\right) \approx \sum_{r=1}^{k k} \alpha_{r}^{(0) i} \varphi_{r}^{i}\left(\zeta_{i}\right) \\
\phi_{i j}\left(\zeta_{i}, \zeta_{j}\right) \approx \sum_{r=1}^{k k}\left[\alpha_{r}^{(i j) i} \varphi_{r}^{i}\left(\zeta_{i}\right)+\alpha_{r}^{(i j) j} \varphi_{r}^{j}\left(\zeta_{j}\right)\right]+\sum_{p=1}^{l} \sum_{q=1}^{l} \beta_{p q}^{(0) i j} \varphi_{p}^{i}\left(\zeta_{i}\right) \varphi_{q}^{j}\left(\zeta_{j}\right)
\end{gathered}
$$

The basis functions of $\phi_{i j}\left(\zeta_{i}, \zeta_{j}\right)$ includes all the basis functions used in $\phi_{i}\left(\zeta_{i}\right)$ and $\phi_{j}\left(\zeta_{j}\right)$. At $N_{\text {samp }}$ sample points of $\zeta$, the HDMR expansions are denoted by the linear algebraic equation system

$$
\eta G=\hat{R}
$$

where $\eta$ is a matrix with size $N_{\text {samp }} \times \tilde{t}$ and all elements of this matrix are basis functions at the $N_{\text {samp }}$ values of $\zeta$; $G$ represents a vector with $\tilde{t}$ dimension of all unknown combination coefficients; $\hat{R}$ denotes a vector 
with $N_{\text {samp }}$-dimension wherein $l$-th element is $\phi\left(\zeta^{(l)}\right)-\phi_{0} \cdot \zeta^{(l)}$ represents the $l$-th sample of $\zeta$, and $\lambda_{0}$ denotes the average value of all $\phi\left(\zeta^{(l)}\right)$. The regression equation for least squares of the above equation can be given as

$$
\frac{1}{N_{\text {samp }}} \eta^{T} \eta G=\frac{1}{N_{\text {samp }}} \eta^{T} \hat{R}
$$

Some rows of the above equation are identical due to the use of extended bases, and an underdetermined algebraic equation system is obtained by removing these rows as

$$
A G=\hat{V}
$$

This has many of solutions for $G$ consisting a manifold $Y \in \mathfrak{R}^{\tilde{t}}$. The main task is to determine a solution $G$ from $Y$ to force the HDMR component functions satisfying the hierarchical orthogonal condition. A solution provided by D-MORPH regression ensures additional condition of exploration path which is denoted by differential equation

$$
\frac{d G(l)}{d l}=\chi v(l)=\left(I_{t}-A^{+} A\right) v(l)
$$

wherein $\chi$ represents orthogonal projector ensuring

$$
\begin{gathered}
\chi^{2}=\chi \quad \text { and } \quad \chi^{T}=\chi \\
\chi=\chi^{2}=\chi^{T} \chi
\end{gathered}
$$

To ensure the wide domain for $G(l)$ and reduce the cost $\kappa(G(l))$, the free function vector is selected and expressed as

$$
v(l)=-\frac{\partial \kappa(G(l))}{\partial G}
$$

Then we obtain

$$
\begin{aligned}
\frac{\partial \kappa(G(l))}{\partial l} & =\left(\frac{\partial \kappa(G(l))}{\partial G}\right)^{T} \frac{\partial G(l)}{\partial l}=\left(\frac{\partial \kappa(G(l))}{\partial G}\right)^{T} P v(l) \\
& =-\left(P \frac{\partial \kappa(G(l))}{\partial G}\right)^{T}\left(P \frac{\partial \kappa(G(l))}{\partial G}\right) \leq 0
\end{aligned}
$$

The cost function is represented in quadratic form as

$$
\kappa=\frac{1}{2} G^{T} B G
$$

where $B$ is the positive definite symmetric matrix and $G_{\infty}$ can be given as

$$
G_{\infty}=V_{t}\left(U_{\tilde{t}-r}^{T} V_{\tilde{t}-r}\right)^{-1} U_{\tilde{t}-r}^{T} A^{+} \hat{V}
$$

where the last columns $(\tilde{t}-r)$ of $U$ and $V$ are denoted as $U_{\tilde{t}-r}$ and $V_{\tilde{t}-r}$, found by decomposition of $\chi B$ 


$$
\chi B=U\left[\begin{array}{ll}
\bar{\zeta}_{r} & 0 \\
0 & 0
\end{array}\right] V^{T}
$$

This unique solution $G_{\infty}$ in $Y$ shows the minimized cost function. The solution ' $G$ ' is determined by using the

D-MORPH to ensure the HDMR component functions' orthogonality in hierarchical manner. In previous literature, construction of the corresponding cost function $\kappa$ can be found [65].

\section{Acknowledgement}

PKK would like to acknowledge the financial support received from MHRD, India during the period of this research work.

\section{References}

[1] Qatu M.S., Leissa A.W., Natural frequencies for cantilevered doubly curved laminated composite shallow shells, Composite Structures, 17, 227-255, 1991.

[2] Dey S., Karmakar A., Natural frequencies of delaminated composite rotating conical shells - A finite element approach, Finite Elements in Analysis and Design, 56, 41-51, 2012.

[3] Haldar S., Sheikh A.H., Bending analysis of composite folded plates by finite element method, Finite Element in Analysis and Design, 47, 477-485, 2011.

[4] Grover N., Singh B.N., Maiti D.K., An efficient $C_{0}$ finite element modeling of an inverse hyperbolic shear deformation theory for the flexural and stability analysis of laminated composite and sandwich plates, Finite Elements in Analysis and Design, 80, 11-22, 2014.

[5] Ye T., Jin G., Zhang Y., Vibrations of composite laminated doubly-curved shells of revolution with elastic restraints including shear deformation, rotary inertia and initial curvature, Composite Structures, 133, 202$225,2015$.

[6] Singh V.K., Panda S.K., Nonlinear free vibration analysis of single/ doubly curved composite shallow shell panels, Thin-Walled Structures, 85, 341-349, 2014.

[7] Panda S.K., Singh, B.N., Large amplitude free vibration analysis of thermally pot-buckled composite doubly curved panel embedded with SMA fibres, Nonlinear Dynamics, 74(1-2), 395-418, 2013.

[8] Versino D., Gherlone M., Di Sciuva M., Four-node shell element for doubly curved multilayered composites based on the refined zigzag theory, Composite Structures, 118, 392-402, 2014.

[9] Fang C., Springer G. S., Design of composite laminates by a Monte Carlo method, Composite Materials, 27(7), 721-753, 1993.

[10] Roy P., Deepu S.P, Pathrikar A., Roy D., Reddy J.N., Phase field based peridynamics damage model for delamination of composite structures, Composite Structures, 180, 972-993, 2017.

[11] Sallam S., G.J. Simitses, Delamination buckling and growth of flat, cross-ply laminates, Composite Structure, 4(4), 361-381, 1985.

[12] Shen M.H.H, Grady J.E., Free vibrations of delaminated beams, AIAA Journal, 30(5):1361-70, 1992.

[13] $\mathrm{Xu}$ Luo-Yu, Interaction between matrix cracking and edge delamination in composite laminates, Composites Science and Technology, 50(4), 469-478, 1994.

[14] Takeda N., Ogihara S., Initiation and growth of delamination from the tips of transverse cracks in CFRP cross-ply laminates, Compos. Sci. Technol., 52, 309-318, 1994.

[15] Sekine H, Natsume T, Fukunaga H. Impact response analysis of partially delaminated composite laminates. JSME Int J., 63(608A), 787-93, 1997.

[16] Hu N., Sekine H., Fukunaga H., Yao Z.H., Impact analysis of composite laminates with multiple delaminations. Int J Impact Eng., 22, 633-48, 1999.

[17] Berthelot J.M., Le Corre J.F., Statistical analysis of the progression of transverse cracking and delamination in cross-ply laminates, Compos. Sci. Technol., 60(14), 2659-2669, 2000. 
[18] Parhi P.K., Bhattacharyya S.K., Sinha P.K., Failure analysis of multiple delaminated due to bending and impact, Bull Mater Sci Indian Acad Sci., 24(2), 143-149, 2001.

[19] Todoroki A., Y. Tanaka, Y. Shimamura, Delamination monitoring of graphite/epoxy laminated composite plate of electric resistance change method, Compos. Sci. Technol., 62(9), 1151-1160, 2002.

[20] Kamiński M., Mechanical and thermal fatigue delamination of curved layered composites, Computers \& Structures, 81(18-19), 1865-1873, 2003.

[21] Rebiere J.L., Gamby D., A criterion for modeling initiation and propagation of matrix cracking and delamination in cross-ply laminates, Compos. Sci. Technol., 64(13-14), 2239-2250, 2004.

[22] Mariani S., Corigliano A., Impact induced composite delamination: state and parameter identification via joint and dual extended Kalman filters, Computer Methods in Applied Mechanics and Engineering, 194(50-52), 5242-5272, 2005.

[23] Karmakar A, Kishimoto K., Free vibration analysis of delaminated composite, JSME Int J, Series A, 49(4), 492-502, 2006.

[24] Chen H., Wang M., Bai R., The effect of nonlinear contact upon natural frequency of delaminated stiffened composite plate, Composite Structures, 76(1-2), 28-33, 2006.

[25] Aymerich F., Dore F., Priolo P., Simulation of multiple delamination in impacted cross-ply laminates using a finite element model based on cohesive interface elements, J. Compos Sci Technol., 69(11-12), 1699-709, 2009.

[26] Wimmer G., Kitzmüller W., Pinter G., Wettemann T., Pettermann H.E., Computational and experimental investigation of delamination in L-shaped laminated composite components, Engineering Fracture Mechanics, 76(18), 2810-2820, 2009.

[27] Kamiński M., Stochastic Boundary Element Method analysis of the interface defects in composite materials, Composite Structures, 94(2), 394-402, 2012.

[28] Shanmugam V., Penmetsa R., Tuegel E., Clay S., Stochastic modeling of delamination growth in unidirectional composite DCB specimens using cohesive zone models, Composite Structures, 102, 38-60, 2013.

[29] Ihesiulor O.K., Shankar K., Zhang Z., Ray T., Delamination detection with error and noise polluted natural frequencies using computational intelligence concepts, Composites Part B: Engineering, 56, 906925, 2014.

[30] Keshava Kumar S., Cinefra M., Carrera E., Ganguli R., Harursampath D., Finite element analysis of free vibration of the delaminated composite plate with variable kinematic multilayered plate elements, Composites Part B: Engineering, 66, 453-465, 2014.

[31] Rama Mohan Rao A., Lakshmi K., Krishna Kumar S., Detection of delamination in laminated composites with limited measurements combining PCA and dynamic QPSO, Advances in Engineering Software, 86, 85-106, 2015.

[32] Iarve E.V., Mollenhauer D.H., Mesh-independent matrix cracking and delamination modeling in advanced composite materials, Numerical Modelling of Failure in Advanced Composite Materials, 227264, 2015.

[33] Zhang, Z., He, M., Liu, A., Singh, H.K., Ramakrishnan, K.R., Hui, D., Shankar, K. and Morozov, E.V., (2018) VIBRATION-BASED assessment of delaminations in FRP composite plates. Composites Part B: Engineering, DOI: 10.1016/j.compositesb.2018.03.003

[34] Sreehari, V. M., \& Maiti, D. K. (2017). Passive control of damaged composite laminates with optimized location of piezoelectric fiber composite patches. Composite Structures, 173, 228-241.

[35] Zheng, W., \& Kassapoglou, C. (2017). Prediction of delamination onset and growth for AP-PLY composite laminates using the finite element method. Composites Part A: Applied Science and Manufacturing, 101, 381-393

[36] Liu, Y., \& Zhang, C. (2018). A critical plane-based model for mixed-mode delamination growth rate prediction under fatigue cyclic loadings. Composites Part B: Engineering, 139, 185-194.

[37] Tornabene, F., Fantuzzi, N., Bacciocchi, M., \& Viola, E. (2018). Mechanical behavior of damaged laminated composites plates and shells: Higher-order Shear Deformation Theories. Composite Structures, 189, 304-329.

[38] Hirwani, C. K., Panda, S. K., \& Mahapatra, T. R. (2018). Nonlinear Finite Element Analysis of Transient Behavior of Delaminated Composite Plate. Journal of Vibration and Acoustics, 140(2), 021001 
[39] Dimitri, R., Tornabene, F., \& Zavarise, G. (2017). Analytical and numerical modeling of the mixedmode delamination process for composite moment-loaded double cantilever beams. Composite Structures.

[40] Scarth, C., \& Cooper, J. E. (2017). Reliability-based aeroelastic design of composite plate wings using a stability margin. Structural and Multidisciplinary Optimization, 1-15.

[41] Grover, N., Sahoo, R., Singh, B. N., \& Maiti, D. K. (2017). Influence of parametric uncertainties on the deflection statistics of general laminated composite and sandwich plates. Composite Structures, 171, 158169.

[42] Chakraborty S., Mandal B., Chowdhury R., Chakrabarti A., Stochastic free vibration analysis of laminated composite plates using polynomial correlated function expansion, Composite Structures, 135, 236-249, 2016.

[43] Lal A., Palekar S.P., Mulani S.B., Kapania R.K., Stochastic extended finite element implementation for fracture analysis of laminated composite plate with a central crack, Aerospace Science and Technology, 60, 131-151, 2017.

[44] Babu A.A., Vasudevan R., Vibration analysis of rotating delaminated non-uniform composite plates, Aerospace Science and Technology, 60, 172-182, 2017.

[45] Papadopoulos V., Seventekidis P., Sotiropoulos G., Stochastic multiscale modeling of graphene reinforced composites, Engineering Structures, 145, 176-189, 2017.

[46] Shaker A., Abdelrahman W. G., Tawfik M., Sadek E., Stochastic finite element analysis of the free vibration of laminated composite plates, Computational Mechanics, 41, 495-501, 2008.

[47] Scarth C., Cooper J.E., Weaver P.M., Silva Gustavo H.C., Uncertainty quantification of aeroelastic stability of composite plate wings using lamination parameters, Composite Structures, 116, 84-93, 2014.

[48] Dey S., Mukhopadhyay T., Adhikari S. (2017) Metamodel based high-fidelity stochastic analysis of composite laminates: A concise review with critical comparative assessment, Composite Structures, 171 227-250

[49] Dey S., Mukhopadhyay T., Spickenheuer A., Gohs U., Adhikari S. (2016) Uncertainty quantification in natural frequency of composite plates - An Artificial neural network based approach, Advanced Composites Letters, 25(2) 43-48

[50] Mukhopadhyay T., Adhikari S. (2016) Free vibration analysis of sandwich panels with randomly irregular honeycomb core, Journal of Engineering Mechanics, 142(11) 06016008

[51] Dey S., Mukhopadhyay T., Naskar S., Dey T. K., Chalak H. D., Adhikari S., Probabilistic characterization for dynamics and stability of laminated soft core sandwich plates, Journal of Sandwich Structures \& Materials, DOI: 10.1177/1099636217694229

[52] Dey T.K., Mukhopadhyay T., Chakrabarti A., Sharma U.K.(2015) Efficient lightweight design of FRP bridge deck, Proceedings of the Institution of Civil Engineers - Structures and Buildings, 168 (10) 697 707

[53] Dey S., Mukhopadhyay T., Khodaparast H. H., Adhikari S. (2016) A response surface modelling approach for resonance driven reliability based optimization of composite shells, Periodica Polytechnica - Civil Engineering, 60 (1) 103-111

[54] Karsh P. K., Mukhopadhyay T., Dey S. (2018) Spatial vulnerability analysis for the first ply failure strength of composite laminates including effect of delamination, Composite Structures, 184 554-567

[55] Dey S., Mukhopadhyay T., Adhikari S. (2018) Uncertainty quantification in laminated composites: A meta-model based approach, CRC Press, Taylor \& Francis Group, ISBN: 9781498784450

[56] Karsh P. K., Mukhopadhyay T., Dey S. (2018) Stochastic dynamic analysis of twisted functionally graded plates, Composites Part B: Engineering, 147 259-278

[57] Naskar S., Mukhopadhyay T., Sriramula S. (2018) Probabilistic micromechanical spatial variability quantification in laminated composites, Composites Part B: Engineering, 151 291-325

[58] Naskar S., Mukhopadhyay T., Sriramula S., Adhikari S. (2017) Stochastic natural frequency analysis of damaged thin-walled laminated composite beams with uncertainty in micromechanical properties, Composite Structures, 160 312-334

[59] Mukhopadhyay T., Naskar S., Dey S., Adhikari S. (2016) On quantifying the effect of noise in surrogate based stochastic free vibration analysis of laminated composite shallow shells, Composite Structures, 140 798-805 
[60] Mukhopadhyay T., Mahata A., Dey S., Adhikari S. (2016) Probabilistic analysis and design of HCP nanowires: an efficient surrogate based molecular dynamics simulation approach, Journal of Materials Science \& Technology, 32(12) 1345-1351

[61] Mahata A., Mukhopadhyay T., Adhikari S. (2016) A polynomial chaos expansion based molecular dynamics study for probabilistic strength analysis of nano-twinned copper, Materials Research Express, 3 036501

[62] Metya S., Mukhopadhyay T., Adhikari S., Bhattacharya G. (2017) System Reliability Analysis of Soil Slopes with General Slip Surfaces Using Multivariate Adaptive Regression Splines, Computers and Geotechnics, 87 212-228

[63] Maharshi K., Mukhopadhyay T., Roy B., Roy L., Dey S. (2018) Stochastic dynamic behaviour of hydrodynamic journal bearings including the effect of surface roughness, International Journal of Mechanical Sciences, 142-143 370-383

[64]Dey S., Mukhopadhyay T., Sahu S. K., Adhikari S. (2018) Stochastic dynamic stability analysis of composite curved panels subjected to non-uniform partial edge loading, European Journal of Mechanics / A Solids, 67 108-122

[65] Li G., Rabitz H., General formulation of HDMR component functions with independent and correlated variables, J. Math Chem., 50, 99-130, 2012.

[66]Mukhopadhyay T., Chowdhury R., Chakrabarti A. (2016) Structural damage identification: A random sampling-high dimensional model representation approach, Advances in Structural Engineering, 19(6) 908-927

[67] Mukhopadhyay T. (2018) A multivariate adaptive regression splines based damage identification methodology for web core composite bridges including the effect of noise, Journal of Sandwich Structures \& Materials, doi.org/10.1177/1099636216682533

[68] Kollar L., Springer G.S., Mechanics of composite structures, Cambridge University Press, 2003.

[69] Meirovitch L. Dynamics and control of structures. NY: John Wiley \& Sons; 1992.

[70] Bathe, K.J., Finite Element Procedures in Engineering Analysis, PHI, New Delhi, 1990, India.

[71] Gim, C. K., Plate finite element modeling of laminated plates. Computers \& Structures, 52(1), 157-168, 1994.

[72] Krawczuk M., Ostachowicz W., Zak A., Dynamics of Cracked Composite Material Structures, Journal of Computational Mechanics 20, 79-83, 1997.

[73] Qatu, M. S., \& Leissa, A. W. (1991). Vibration studies for laminated composite twisted cantilever plates. International Journal of Mechanical Sciences, 33(11), 927-940

[74] Mukhopadhyay T., Adhikari S. (2017) Stochastic mechanics of metamaterials, Composite Structures, $16285-97$

[75] Mukhopadhyay T., Mahata A., Adhikari S., Asle Zaeem M. (2017) Effective elastic properties of two dimensional multiplanar hexagonal nano-structures, 2D Materials, 4025006

[76] Mukhopadhyay T., Adhikari S. (2017) Effective in-plane elastic moduli of quasi-random spatially irregular hexagonal lattices, International Journal of Engineering Science, 119 142-179

[77] Mukhopadhyay T., Adhikari S., Batou A., Frequency domain homogenization for the viscoelastic properties of spatially correlated quasi-periodic lattices, International Journal of Mechanical Sciences, DOI: 10.1016/j.ijmecsci.2017.09.004

[78] Mukhopadhyay T., Mahata A., Adhikari S., Asle Zaeem M. (2017) Effective mechanical properties of multilayer nano-heterostructures, Scientific Reports, 715818

[79] Mukhopadhyay T., Mahata A., Adhikari S., Asle Zaeem M. (2018) Probing the shear modulus of twodimensional multiplanar nanostructures and heterostructures, Nanoscale, 105280 - 5294

[80] Mukhopadhyay T. (2017) Mechanics of quasi-periodic lattices, PhD Thesis, Swansea University

[81] Dey S., Mukhopadhyay T., Adhikari S.(2015) Stochastic free vibration analyses of composite doubly curved shells - A Kriging model approach, Composites Part B: Engineering, 70 99-112

[82] Deutsch F., Best approximation in inner product space, Springer, New York, 2000.

[83] Li G., Wang S.W., Rabitz H., Wang S., Jáffe P.R., Global uncertainty assessments by high dimensional model representations (HDMR), Chem. Eng. Sci., 57, 4445-4460, 2002.

[84] Wang S.W., Levy H. II, Li G., Rabitz H., Fully equivalent operational models for atmospheric chemical kinetics within global chemistry-transport models, J. Geophys. Res., 104(D23), 30417-30426, 1999. 
[85] Li G., Hu J., Wang S.W., Georgopoulos P.G., Schoendorf J., Rabitz H., Random sampling-high dimensional model representation (RS-HDMR) and orthogonality of its different order component functions, J. Phys. Chem. A, 110, 2474-2485, 2006. 